The FASEB Journal express article 10.1096/fj.04-2529fje. Published online January 27, 2005.

\title{
Endothelial cell modulation of bone marrow stromal cell osteogenic potential
}

\author{
Darnell Kaigler, ${ }^{*}$ Paul H. Krebsbach, ${ }^{\dagger}$ Erin R. West, ${ }^{\dagger}$ Kim Horger, ${ }^{\ddagger}$ Yen-Chen Huang, ${ }^{\ddagger}$ and \\ David J. Mooney ${ }^{\S}$
}

Departments of *Biologic and Materials Sciences, ${ }^{\dagger}$ Oral Medicine/Pathology/Oncology, and Chemical and Biomedical Engineering, University of Michigan, Ann Arbor, Michigan 48109, USA; and ${ }^{\S}$ Division of Engineering and Applied Sciences, Harvard University, Cambridge, Massachusetts 02138, USA

Corresponding author: David J. Mooney, Division of Engineering and Applied Sciences, Harvard University, 29 Oxford Street, 325 Pierce Hall, Cambridge, MA 02138, USA. E-mail: mooneyd@deas.harvard.edu

\section{ABSTRACT}

In the context of bone development and regeneration, the intimate association of the vascular endothelium with osteogenic cells suggests that endothelial cells (ECs) may directly regulate the differentiation of osteoprogenitor cells. To investigate this question, bone marrow stromal cells (BMSCs) were cultured: in the presence of EC-conditioned medium, on EC extracellular matrix, and in EC cocultures with and without cell contact. RNA and protein were isolated from ECs and analyzed by reverse transcriptase-polymerase chain reaction and Western blotting, respectively, for expression of bone morphogenetic protein 2 (BMP-2). In animal studies, BMSCs and ECs were cotransplanted into severe combined immunodeficient mice on biodegradable polymer matrices, and histomorphometric analysis was performed to determine the extent of new bone and blood vessel formation. ECs significantly increased BMSC osteogenic differentiation in vitro only when cultured in direct contact. ECs expressed BMP-2, and experiments employing interfering RNA inhibition confirmed its production as contributing to the increased BMSC osteogenic differentiation. In vivo, cotransplantation of ECs with BMSCs resulted in greater bone formation than did transplantation of BMSCs alone. These data suggest that ECs function not only to form the microvasculature that delivers nutrients to developing bone but also to modulate the differentiation of osteoprogenitor cells in vitro and in vivo.

Key words: bone development $\bullet$ bone regeneration $\bullet$ osteoprogenitor cells $\bullet$ BMP-2 $\bullet$ osteogenic differentiation

$I^{1}$ $\mathrm{t}$ is well-established that the process of new blood vessel formation, or angiogenesis, is coupled to the development and maturation of bone (1). The intimate association of the vascular endothelium with bone and osteogenic cells suggests that endothelial cells (ECs) could be prime sources of modulators of bone development and function. Not only do microvascular ECs line new blood vessels and capillaries that support developing bone tissue, they also produce a number of local and systemic mediators that could influence the recruitment, 
proliferation, differentiation, and function of various target cells (2-5). In different coculture systems, ECs have had direct effects on the osteogenic potential of rat calvarial bone cells $(6,7)$. More recently, gap junctional communication between ECs and osteoprogenitor cells has been reported to be a key mediator in the enhancement of osteogenic activity of osteoprogenitor cells (8). There have also been reports of expression of bone morphogentic protein 2 (BMP-2) by bone marrow ECs (9) and microvascular ECs (10); however, the functional significance of the presence of BMP-2 in ECs remains unclear in the context of bone formation.

The goal of this study was to determine whether ECs can directly modulate the osteogenic potential of osteoprogenitor cells in vitro and in vivo. Multipotent cells derived from bone marrow are a popular cell type to study the differentiation of osteogenic cells and bone development, because of their role in regeneration and potential applications in tissue engineering. Colonies formed from a single precursor cell from marrow are termed colonyforming unit fibroblasts $(11,12)$, and the progeny of these fibroblasts have been defined as bone marrow stromal cells (BMSCs). These BMSCs represent a heterogeneous cell population containing cells capable of extensive proliferation and differentiation into several cell types, including osteoblasts, chondrocytes, fibroblasts, adipocytes, and cells forming the hematopoiesis-supporting reticular stroma $(11,13-15)$. Because of their osteogenic potential, BMSCs have been used in a number of different systems employing therapeutic strategies of tissue regeneration and engineering (16-19). It is for these reasons that BMSCs were used to represent an osteoprogenitor cell type in these studies.

The hypothesis underlying the current studies is that ECs have the ability to directly enhance the osteogenic potential of BMSCs. To test this hypothesis, the influence of ECs on BMSC osteogenic differentiation was examined in different in vitro coculture systems. After we found that ECs enhanced BMSC osteogenic differentiation and produced BMP-2, the role of BMP-2 in BMSC osteogenic differentiation was elucidated. Finally, ECs and BMSCs were cotransplanted on biodegradable polymer scaffolds to determine whether ECs affect bone formation in vivo in a manner consistent with their enhancement of BMSC osteogenic differentiation in vitro.

\section{MATERIALS AND METHODS}

\section{Cell culture}

\section{Culture of ECs}

Human dermal microvascular ECs (HDMECs) were purchased (Cambrex BioScience, Walkersville, MD) and cultured in microvascular endothelial cell medium 2 (EGM2-MV) containing 5\% FBS (Cambrex BioScience). EC-conditioned medium (EC CM) was collected after $12 \mathrm{~h}$ of cell exposure and concentrated 10-fold by centrifugation with an UltraFree-15 centrifugal filter device, which had a membrane with a nominal molecular size limit of $5 \mathrm{kDa}$ (Millipore, Bedford MA). Aliquots of the CM were frozen until ready to use in BMSC cultures. When ECs reached $85-90 \%$ confluence, they were subcultured: in 12-well plates to condition wells with their extracellular matrix (ECM), onto 12-well Transwell inserts at a density of $2 \times$ $10^{4}$ cells/insert, or in 12-well plates to grow in coculture with BMSCs. Cells that were seeded for production of ECM were removed from wells with $2 \mathrm{M}$ urea in EGM2-MV 3 days after seeding, after which plates were used immediately for culturing BMSCs. 


\section{Culture of BMSCs}

Human bone marrow was collected from patients undergoing iliac bone graft procedures (with University of Michigan Institutional Review Board approval), and BMSCs were isolated as described previously $(20,21)$. Briefly, marrow was placed in ice-cold modified minimum essential medium ( $\alpha$ MEM) (Life Technologies, Grand Island, NY) with $100 \mathrm{U} / \mathrm{ml}$ sodium heparin (Fisher Scientific, Fair Lawn, NJ) and was centrifuged at $700 \mathrm{~g}$ for $10 \mathrm{~min}$; the cell pellet was then resuspended in fresh $\alpha$ MEM. All preparations were pipetted repeatedly to break up cell aggregates. Subsequently, marrow cell suspensions were passed consecutively through 16.5 - and 20.5-gauge needles before culture. Cells were cultured in $\alpha$ MEM, $2 \mathrm{mM}$ glutamine, $100 \mathrm{U} / \mathrm{ml}$ penicillin, $100 \mu \mathrm{g} / \mathrm{ml}$ streptomycin sulfate (Biofluids, Rockville, MD), and 10\% FBS (Life Technologies). BMSCs were then subcultured in T-75 tissue culture flasks in aMEM containing $1 \%$ penicillin/streptomycin and 10\% FBS. Once cells reached $85-90 \%$ confluence, they were subcultured in 12-well plates or 12-well Transwell plates at a density of $1 \times 10^{4} \mathrm{cells} / \mathrm{cm}^{2}$.

Cells were then cultured in the presence of EC CM, on EC ECM, in the presence of ECs cultured on the inserts of 12-well Transwell plates, or in coculture with various ratios of ECs to BMSCs. BMSCs were also cocultured at 1:1 ratios with a human foreskin fibroblast (HFF) cell line (HFF1; American Type Culture Collection, Manassas, VA) and with primary human gingival fibroblasts (HGF) extracted from excess gingival tissue from dental surgical procedures at the University of Michigan with Institutional Review Board approval. These conditions both served as control groups. The medium used in all coculture experiments was a 50:50 mixture of EC culture medium (EGM2-MV) and BMSC culture medium ( $\alpha$ MEM containing $1 \%$ penicillin/streptomycin and 10\% FBS). The 50:50 mixture was used in the coculture experiments so as to minimize the amount of exogenous growth factors (contained in EGM2-MV) in these experiments, while maintaining enough factors in the medium to sustain the viability of the ECs. BMSCs were cultured from 3 to 14 days, depending on the time course of the experiment. All studies used BMSCs below their ninth passage in culture.

\section{Analysis of osteogenesis in vitro}

\section{Alkaline phosphatase assays}

Alkaline phosphatase (ALP) assays were performed to measure early osteoblastic differentiation of BMSCs. Cells were lysed by using passive lysis buffer (Promega, Madison, WI), and lysates were sonicated for 4-5 s and transferred to ice. Samples were centrifuged for $10 \mathrm{~min}$, and $100 \mu \mathrm{l}$ of the supernatant was collected. The remaining volume of supernatant and lysate was used for DNA analysis (Hoescht Dye Assay, Sigma-Aldrich, St. Louis, MO). ALP activity in samples was quantified by means of a colorimetric assay as described previously (22). ALP activity values were normalized to the amount of BMSC DNA, not total cellular DNA in a sample, to obtain units of ALP/mg DNA. Staining was also performed for ALP with cells fixed in $70 \%$ EtOH, as described previously (23). Four independent coculture experiments were performed.

\section{Cell number normalization in cocultures}

Total DNA was collected from samples and quantified by the Hoescht Dye Assay (24). In coculture experiments, the number of BMSCs was determined to allow normalization of ALP 
and osteocalcin levels to BMSC cell number, instead of total cell number, to account for the two cell types in coculture assays. To determine the ratio of ECs to BMSC in these coculture DNA samples, two measures were used. First, staining with acetylated low-density lipoprotein, labeled with 1,1'-dioctadecyl-3,3,3',3'-tetramethylindocarbocyanine perchlorate (DiI-Ac-LDL; Biomedical Technologies, Stoughton, MA), was performed in Lab-Tek chamber slides (NalgeNunc International, Rochester, NY) on parallel cultures to determine qualitatively the contribution of ECs to total cell number in cocultures. DiI-Ac-LDL was diluted to $10 \mu \mathrm{g} / \mathrm{ml}$ in EGM2-MV, added to cocultures, and allowed to incubate for $4 \mathrm{~h}$ at $37^{\circ} \mathrm{C}$. Medium was then removed and cultures were rinsed three times with PBS. Cells were then fixed in 3\% formaldehyde in PBS for $20 \mathrm{~min}$ at room temperature. After fixation, cells were rinsed in deionized water and excess liquid was drained. Wells were removed from Lab-Tek chamber slides and covered with coverslips, with $90 \%$ glycerol and $10 \%$ PBS, and the numbers of positive and total cells were counted.

For quantitative measures of EC numbers in cocultures, ECs were isolated from the total mix of cells in cocultures and counted by using the EasySep protocol (StemCell Technologies, Vancouver, British Columbia, Canada). All cells were removed from tissue culture plates with trypsin $(0.05 \%)$, pelleted, and resuspended in PBS. EasySep CD31 cocktail (15 $\mu \mathrm{g}$ of anti-human CD31; DakoCytomation, Carpinteria, CA) was added to samples maintained at $37^{\circ} \mathrm{C}$ for $15 \mathrm{~min}$. EasySep Magnetic Nanoparticles were next added to samples and incubated for $10 \mathrm{~min}$. The sample volumes were increased to $2.5 \mathrm{ml}$ with PBS, and the EasySep magnet was used to separate ECs from the total cell population. The ECs were subsequently counted with a model ZM Coulter Counter (Beckman Coulter, Fullerton, CA). The number of ECs was subtracted from the total cell number to determine the percentage of BMSCs and ECs in cocultures. The data obtained from these assays were used to normalize the ALP levels.

\section{Osteocalcin ELISA}

A Mid-Tact Human Osteocalcin (OCN) ELISA Kit (Biomedical Technologies) was used for the detection of human OCN secreted by BMSCs. OCN is a late-stage osteogenic differentiation marker. BMSCs were grown alone or in coculture with ECs for 14 days. Medium was changed 1 day after cell seeding and every 2 days thereafter. Seven and 14 days after cell seeding, 12-h CM was collected from both culture conditions and measured for the presence of OCN. Three independent coculture experiments were performed.

\section{BMP-2 expression in ECs}

\section{$B M P-2$ reverse transcription-polymerase chain reaction ( $R T-P C R)$}

RNA was isolated from HDMECs with the TRIzol method (TRIzol Reagent; Invitrogen, Carlsbad, CA) and used for the generation of HDMEC cDNA. To generate HDMEC cDNA, RNA first underwent RT with SuperScript II RNase H Reverse Transcriptase (Invitrogen), with oligo(dT) to hybridize to 3'-poly(A) tails. After generation of cDNA, the target sequence was amplified with a PCR by using BMP-2 primers: 5'-ACG CGG GAG AAG GAG GAG G-3' (175-193) and 3'-TGT TAT CGT CAA AGG TAG TGG-5' (881-831). PCR products were generated with Taq DNA polymerase in a thermocycler (GeneAmp PCR System 2400; PerkinElmer, Boston, MA) under the following conditions: $95^{\circ} \mathrm{C}$ for 2 min to denature cDNA, $94^{\circ} \mathrm{C}$ for 
$30 \mathrm{~s}, 58^{\circ} \mathrm{C}$ for $30 \mathrm{~s}$, and $72^{\circ} \mathrm{C}$ for $2 \mathrm{~min}$, for a total of 35 cycles plus a final $10 \mathrm{~min}$ at $72^{\circ} \mathrm{C}$. A sample of $1 \mu \mathrm{l}$ of the PCR products was loaded onto $1 \%$ agarose gel and analyzed via electrophoresis.

\section{Western blot analysis}

Cell lysates were collected from ECs with incubation for $5 \mathrm{~min}$ in a simple lysis buffer ( $25 \mathrm{mM}$ Tris-Cl, $\mathrm{pH} 7.4,0.4 \mathrm{NaCl}, 0.5 \%$ sodium dodecyl sulfate; all reagents were from SigmaAldrich), followed by scraping of the cell monolayer to enhance protein dissolution. Lysates were frozen at $-20^{\circ} \mathrm{C}$ until used. Total protein was determined by using the Bio-Rad protein assay; equivalent concentrations of total protein were diluted at 1:2 ratios with $2 \times$ Laemmli sample buffer (Bio-Rad, Hercules, CA), boiled for $5 \mathrm{~min}$ to reduce protein, and loaded onto 10$20 \%$ Tris- $\mathrm{HCl}$ polyacrylamide gels (Bio-Rad). Gels were electrophoresed for $42 \mathrm{~min}$ at $200 \mathrm{~V}$. Polyvinylidine difluoride membranes (Bio-Rad) were used for blotting, and protein was electrophoretically transferred via the Mini Trans-Blot system (Bio-Rad) for $1.5 \mathrm{~h}$ at $100 \mathrm{~V}$. After transfer, nonspecific protein binding was blocked by incubating blots with 5\% BSA in TBS (Tris-buffered saline: $10 \mathrm{mM}$ Tris, $\mathrm{pH} 7.5,100 \mathrm{mM} \mathrm{NaCl}$ ) containing $0.1 \%$ Tween-20 (TBS-T) overnight at $4{ }^{\circ} \mathrm{C}$. After blocking, blots were rinsed three times for 5 min each time with TBS-T with rocking. Blots were then incubated for $1 \mathrm{~h}$ with a primary monoclonal anti-human BMP-2 antibody (R\&D Systems, Minneapolis, MN) diluted 1:1000 in blocking solution. After three more rinses with TBS-T, blots were incubated for $1 \mathrm{~h}$ with a secondary goat anti-mouse horseradish peroxidase (HRP)-conjugated antibody (Bio-Rad) diluted 1:1000. Signal detection was achieved with the ECL detection system (Amersham Biosciences, Piscataway, NJ), and signals were recorded on film (Hyperfilm; Amersham Biosciences).

\section{BMP-2 silencing with small interfering RNA (siRNA)}

\section{Generation of BMP-2 siRNA}

RNA was isolated from HDMECs with the TRIzol method and used for the generation of BMP-2 siRNA via the Invitrogen system. PCR products generated in the manner outlined above (RTPCR) were joined to the BLOCK-iT T7-TOPO Linker through the linking reaction. After this reaction was completed, a secondary amplification of the PCR products was performed to generate the sense and antisense linear DNA templates. Two amplification reactions were set up, one with BLOCK-iT T7 Primer and BMP-2 3'-TGT TAT CGT CAA AGG TAG TGG-5', the other with BLOCK-iT T7 Primer and 5'-ACG CGG GAG AAG GAG GAG G-3'. DNA templates were amplified in the thermocycler under the same conditions as the initial PCR reaction. Secondary amplification reaction products were analyzed by gel electrophoresis. Sense and antisense linear DNA templates generated from this reaction were transcribed by using the BLOCK-iT T7 Enzyme Mix to produce sense and antisense single-stranded RNA transcripts. RNA transcripts were then purified and annealed to produce double-stranded RNA (dsRNA). The BMP-2 dsRNA was next purified and diced into 21-23 nucleotide fragments (d-siRNA) by the BLOCK-iT Dicer Enzyme. The d-siRNA was purified, placed into 3- $\mu$ l aliquots (460 ng/ $\mu \mathrm{l})$, and stored at $-70^{\circ} \mathrm{C}$ until ready to use for transfection. 


\section{BMP-2 siRNA transfections}

HDMECs were plated in 12-well dishes and allowed to attach for $24 \mathrm{~h}$. Cells were then rinsed $1 \times$ with PBS, after which endothelial basal medium-2 (EBM-2) was added to each well. BMP-2 siRNA was mixed with Lipofectamine 2000 in EBM-2 with no antibiotic to make a final working dilution of $700 \mathrm{ng}$ of siRNA/ml of medium (w/v) and $10 \mu \mathrm{l}$ of Lipofectamine 2000/125 $\mu \mathrm{l}$ of medium $(\mathrm{v} / \mathrm{v})$. Dilutions were also made without the addition of siRNA for use as a negative control transfection solution. These solutions were then added to the appropriate wells of the tissue culture plate. Transfection medium was left on cells for $6 \mathrm{~h}$ and then changed to EGM2-MV. At 24 h, 48 h, 72 h, 96 h, and 5 days after transfection, Western blotting was performed to determine BMP-2 protein expression of HDMECs transfected with BMP-2 siRNA. ECs inhibited in their production of BMP-2 (EC-siRNA) (72 $\mathrm{h}$ after transfection) were cocultured with BMSCs. After 2 days in coculture with these ECs, ALP expression of BMSCs was measured. ALP expression was also measured for BMSCs cultured alone and BMSC cocultured with ECs transfected with only the Lipofectamine reagent (EC-Lipo) (control). Three independent coculture experiments were performed.

\section{In vivo cell transplantation}

\section{Poly(lactic co-glycolic acid) (PLGA) scaffold fabrication}

A copolymer of D,L-lactide and glycolide (PLGA) (85:15 molar ratio) (Alkermes, Cambridge, MA) was used in a gas-foaming process (25) to form matrices. Granular PLGA was ground (after being frozen in liquid nitrogen) by using a Science-ware Micro-Mill (Bel-Art Products, Pequannock, NJ) and sieved to obtain particles between 106 and $250 \mu \mathrm{m}$. The $40 \mathrm{mg}$ of PLGA particulate was mixed with $760 \mathrm{mg}$ of $\mathrm{NaCl}$ and loaded into a custom-built cylindrical mold (diameter $=4.763 \mathrm{~mm}$ ) and compressed for $60 \mathrm{~s}$, at $1500 \mathrm{psi}$, in a Carver laboratory press (Carver, Wabash, IN). The matrices were then placed in a pressure vessel and exposed to 800 psi of $\mathrm{CO}_{2}$ gas for $16 \mathrm{~h}$. The pressure was rapidly (less than $2 \mathrm{~min}$ ) released to ambient to cause the polymer particles to foam and ultimately fuse to form a porous polymer matrix. $\mathrm{NaCl}$ was leached with $0.5 \mathrm{M} \mathrm{CaCl}_{2}$ solution for $16 \mathrm{~h}$.

\section{Cell seeding and implantation}

Before cells were seeded, scaffolds (13 $\mathrm{mm}$ in diameter, $3 \mathrm{~mm}$ thick) were cut into quarters and immersed in $100 \% \mathrm{EtOH}$ for $1 \mathrm{~h}$, followed by five rinses of PBS. Just before seeding of cells, polymers were wicked with $2 \times 2$ gauze (Johnson \& Johnson) to remove excess residual PBS. Samples of $50 \mu \mathrm{l}$ of cell suspensions, containing $5 \times 10^{5}$ cells of the appropriate cell type for each condition, were added to each scaffold with a Pipetman. In the BMSC-only condition, $50 \mu 1$ of additional medium was used to equalize the liquid volumes added to each scaffold. Scaffolds were then incubated for $1 \mathrm{~h}$ before implantation. Scaffolds containing the appropriate cells for each of the two conditions were implanted into two subcutaneous pockets on the dorsal region of 7- to 9-wk-old male severe combined immunodeficient (SCID) mice (cb17/SCID) (Taconic Laboratories, Germantown, NY). Two independent implants were placed in each animal, and a total of five implants were placed for each experimental condition per time point. Animals were first anesthetized with an intraperitoneal injection of $1 \mathrm{ml}$ of ketamine $(100 \mathrm{mg} / \mathrm{ml})$ and $0.15 \mathrm{ml}$ of xylazine $(20 \mathrm{mg} / \mathrm{ml})$. After the animal no longer responded to stimuli (e.g., foot pinches), a 2 
$\times 2 \mathrm{~cm}$ area was shaved clean on its back. This exposed skin was then wiped, first with gauze soaked with Betadine (Purdue Frederick, Stamford, CT) followed by gauze soaked with 70\% ethanol (Fisher Scientific). A small (about 1.5-cm) longitudinal incision was then made into the skin at the center of the shaved area. The tips of dissecting scissors were slipped under the skin (between the skin and muscle layers) to bluntly dissect subcutaneous fascial tissue and create pockets. Implants were placed, and the incisions were closed with interrupted sutures. The closed wound was covered with tissue adhesive (Mastisol; Ferndale Laboratories, Ferndale, MI) to help seal the opening and prevent the mice from chewing on the sutures. Animals were allowed to recover postoperatively for $1 \mathrm{~h}$ under a heat lamp before being returned to their respective cages. At 2, 4, and $8 \mathrm{wk}$, animals were killed and implants were analyzed for bone and blood vessel formation.

\section{Analysis of bone and blood vessel formation in vivo}

\section{Tissue preparation and histological staining}

Implants were retrieved at various time points and fixed overnight (about $16 \mathrm{~h}$ ) in aqueous buffered zinc formalin (Z-Fix; Anatech, Battle Creek, MI). Implants were then transferred to $70 \%$ EtOH until they were processed and embedded in paraffin. Paraffin-embedded matrices were cut into serial sections ( $5 \mu \mathrm{m}$ thick) and placed on glass slides for histological analysis. Tissue sections were stained with Gill's 3 hematoxylin (Sigma-Aldrich) and aqueous eosin Y solution (Sigma-Aldrich) (H\&E) to visualize the overall tissue morphology. Von Kossa staining was performed with $5 \%$ silver nitrate solution for $1 \mathrm{~h}$. After rinses in water, sections were placed in photographic developer for $2 \mathrm{~min}$ and, after several additional rinses in water, excess silver nitrate (Sigma-Aldrich) was neutralized with 5\% sodium thiosulfate solution (Sigma-Aldrich). Sections were subsequently counterstained in nuclear fast red solution (Sigma-Aldrich).

Immunostaining of human bone sialoprotein (BSP) and human-derived blood vessels was performed via an anti-human BSP antibody (Chemicon, Temecula, CA) and a specific mouse anti-human CD31 antibody (DakoCytomation), respectively, as described previously (26). Briefly, sections were first deparaffinized with xylene (Sigma-Aldrich), taken through a descending series of EtOH concentrations, rehydrated in $\mathrm{H}_{2} \mathrm{O}$, and then placed in $3 \% \mathrm{H}_{2} \mathrm{O}_{2}$ (Sigma-Aldrich) in PBS to quench endogenous peroxidase activity. To improve antigen exposure, the sections were boiled in citrate buffer (Biocare Medical, Walnut Creek, CA) in a pressure cooker (Biocare Medical) and then incubated with Terminator blocking solution (Biocare Medical). Primary antibodies were diluted in Da Vinci Green antibody diluent (Biocare Medical) at concentrations of 1:1000 (for CD31) or 1:300 (for BSP) and were allowed to incubate overnight at $4^{\circ} \mathrm{C}$. Sections were washed free of primary antibody and incubated with a broad-spectrum biotinylated Universal Link (Biocare Medical) followed by an incubation with streptavidin-HRP (Biocare Medical). The HRP reaction was completed with the addition of 3,3'diaminobenzidine (Zymed, South San Francisco, CA) for $30 \mathrm{~s}$. The slides were counterstained with hematoxylin and finally dehydrated, mounted, and covered with coverslips.

\section{Quantification of blood vessels and bone}

Tissue sections were visualized and photographed with the E-800 light microscope (Nikon, Melville, NY). Five samples from each condition were analyzed manually and digitally. Blood 
vessels present in the implants were analyzed for total number. Blood vessels were identified in H\&E-stained tissues, at $\times 200$ magnification, by defined lumens and the presence of red blood cells within their boundaries. Experimental conditions that included transplanted HDMECs were stained for the presence of human CD31 antigen to allow for the identification and counting of human-derived blood vessels.

Bone histomorphometric analysis of sections stained with H\&E was performed to quantitate bone formation. By means of an E-800 light microscope, histological sections from each implant at each time point were scanned and imported into Adobe Photoshop (Adobe Systems, Mountain View, CA). Bone tissue area for each section was determined by dividing the total number of bone pixels by the total number of implant pixels based on color by using ImagePro Plus software (MediaCybernetics, San Diego, CA). This value was plotted as bone area/tissue area. Identification of bone was based on morphology of eosin-stained tissue and the identification of cells lining (osteoblasts) and within (osteocytes) this tissue. Selected von Kossa- and BSPstained sections were evaluated to verify the presence of bone tissue in the H\&E-stained sections used for analyses.

\section{Statistical analysis}

Statistical analyses were performed with Instat software (Graphpad Software, San Diego, CA). Descriptive analyses were performed initially, followed by the use of Student's $t$ tests or oneway ANOVA, according to the experimental design. Statistical significance was measured as $P<$ 0.05. All data were plotted as means \pm SE. Statistically significant differences in histomorphometric analysis were determined by using one-tailed Student's $t$ tests, and statistical significance was defined by $P<0.05$.

\section{RESULTS}

\section{In vitro studies}

\section{Effects of ECs on BMSC osteogenic differentiation}

To determine the effects of ECs on BMSC osteogenic differentiation, BMSCs were cultured in the presence of EC CM, in medium shared with ECs, on EC ECM, and in coculture with ECs. ALP expression was used as an early measure of osteogenic differentiation. In the presence of EC CM, shared medium, and ECM, there were no significant differences in expression of ALP from BMSCs (data not shown). However, major differences in BMSC ALP activity and expression patterns were observed when BMSCs were cocultured with ECs (Fig. 1A). Quantitative analysis of ALP activity confirmed the qualitative observations, as there was a statistically significant $(P<0.05)$ increase (as much as twofold) in ALP activity in BMSC/EC cocultures compared with BMSCs alone (Fig. 1B). ECs cultured alone and two additional control groups, BMSCs in coculture with HFF and BMSCs in coculture with HGF, were assayed for ALP activity. ECs alone and BMSCs cocultured with HFF showed very minimal baseline levels of ALP. BMSCs cocultured with HGF showed no significant increase in ALP activity (Fig. 1B). Osteogenic differentiation was also enhanced as a function of increased numbers of ECs in cocultures (Fig. 1C). As an additional measure of BMSC osteogenic differentiation in coculture conditions, BMSC expression of a later stage osteogenic marker, OCN, was quantified. OCN is a 
specific product of mature osteoblasts and is primarily deposited into the ECM of bone (27). In 2-wk cocultures of BMSCs and ECs, BMSCs production of OCN was significantly higher than was OCN production from 2-wk cultures of BMSCs alone (Fig. 1D). OCN from ECs was also measured, but no detectable levels were present in EC CM (data not shown).

\section{Role of BMP-2 in EC-induced osteogenic differentiation of BMSCs}

Expression of BMP-2 mRNA from ECs has been reported $(9,10)$, but its functional significance in BMSC osteogenic differentiation has not. In addition, it is unclear whether two additional osteoinductive BMPs (-4 and -7) are produced by ECs. To address these issues, RNA was first isolated from ECs and analyzed for BMP-2, -4, and -7 mRNA with RT-PCR. BMP-4 and BMP-7 were not detected (data not shown), but expression of BMP-2 was detected in HDMECs and in another type of EC, human umbilical vein ECs (HUVECs) (Fig. 2A). HUVECs were analyzed to determine whether BMP-2 expression patterns were specific to HDMECs or common to more than one type of EC. Western blotting of cell lysates from HDMECs and HUVECs was subsequently performed to analyze for BMP-2 protein expression. Blotting confirmed the synthesis of BMP-2 from both HDMECs and HUVECs (Fig. 2B). Additional coculture experiments with BMSCs and ECs were conducted, and Western blot analysis indicated no detectable difference in BMP-2 production by ECs when cultured alone or when cocultured with BMSCs (data not shown).

To determine the role of EC production of BMP-2 in osteogenic differentiation of BMSCs, the production of BMP-2 in ECs was inhibited with BMP-2 siRNA (Fig. 3A). ECs that were inhibited in their production of BMP-2 with siRNA were then cocultured with BMSCs, and osteogenic differentiation of BMSCs, as measured by ALP activity, was determined. A large decrease was found in osteogenic differentiation of BMSCs in cocultures with EC-siRNA compared with BMSCs grown in coculture with EC-Lipo (control coculture condition) (Fig. 3B). Quantitative analysis of ALP activity confirmed a statistically significant reduction in osteogenic differentiation of BMSCs cocultured with EC-siRNA relative to the control coculture condition (Fig. 3C). Additional coculture experiments were performed and showed that the effect of ECs on BMSC ALP activity could be mimicked by adding recombinant human BMP-2 at a concentration of $100 \mathrm{ng} / \mathrm{ml}$ (data not shown). These findings confirm that BMP-2, produced by ECs, plays a role in osteogenic differentiation of BMSCs.

\section{In vivo studies}

\section{Blood vessel analysis: 2 wk}

To determine whether ECs enhance BMSC osteogenic differentiation in vivo, BMSCs were cotransplanted with ECs. Before bone formation was measured, blood vessels were analyzed to determine whether transplanted ECs enhanced neovascularization within the scaffolds. Two weeks after implantation, samples were retrieved and analyzed for neovascularization within the scaffold by means of functional blood vessel counts. There was no significant difference between the total number of functional vessels formed within scaffolds containing both BMSCs and ECs and scaffolds containing only BMSCs (Fig. 4A). Human CD31 immunostaining was performed at this early time point to determine the number of functional vessels that were composed in whole or in part from ECs that were donor derived. Immunostaining clearly displayed vessels 
derived from donor ECs as well as chimeric vessels derived from both host and donor ECs (Fig. $\underline{4 B})$. The percentage of donor EC-derived vessels relative to total vessels increased significantly in scaffolds containing human BMSCs and ECs compared with scaffolds containing BMSCs alone (Fig. 4C).

\section{Blood vessel and bone analysis: $4 \mathrm{wk}$}

Bone formation and neovascularization within the scaffolds were also measured at 4 wk posttransplantation. As at $2 \mathrm{wk}$, there was no significant difference between the total number of vessels formed within scaffolds containing both BMSCs and ECs compared with scaffolds containing only BMSCs (Fig. 5A); BMSCs alone produced a potent angiogenic response (Fig. $\underline{5 B})$. Histological examination revealed localized regions of bony islands in both conditions. Comparison of bone tissue histomorphometry from these scaffolds revealed a $7 \%$ increase in bone formation in scaffolds containing human BMSCs and ECs relative to scaffolds containing BMSCs alone (Fig. 5C). This increase in bone formation was not statistically significant, however $(P<0.1)$.

\section{Blood vessel and bone analysis: 8 wk}

Blood vessel and bone analysis was also performed at $8 \mathrm{wk}$ post-transplantation. There was no significant difference between the total number of functional blood vessels formed within scaffolds containing both BMSCs and ECs and scaffolds containing only BMSCs (Fig. 6A). A significantly higher number of vessels were donor derived in scaffolds containing human BMSCs and ECs than in scaffolds containing BMSCs alone (ㅍi. $6 B$ ).

In the evaluation of bone tissue formation, gross qualitative histological analysis of whole sections suggested that scaffolds cotransplanted with BMSCs and ECs formed more bone tissue than scaffolds transplanted with BMSCs alone (i․ $\underline{\text { FA }}$ ). Bone tissue had a wavy, trabecular appearance with little to no presence of hematopoietic tissue formation. Representative sections were also immunostained for a bone tissue-specific marker, BSP, and the positive staining gave further evidence for the presence of osteoblasts and bone-like tissue formation (Fig. 7B). The formation of a mineralized ECM, indicative of bone formation, was confirmed with von Kossa staining of additional sections (Fig. 7C). Quantitative bone histomorphometry revealed a statistically significant $14 \%$ increase in engineered bone formation in implants containing both cell types compared with implants containing only BMSCs (i․ 7D).

\section{DISCUSSION}

These studies demonstrated that ECs enhance the osteogenic potential of BMSCs when grown in coculture in vitro. These results confirmed that ECs produce BMP-2, a potent bone morphogen, and indicated the functional importance of this BMP-2 in the osteogenic differentiation of BMSCs. Finally, in vivo, transplanted ECs enhance the bone-forming capacity of transplanted BMSCs.

The results of these studies indicate that BMSC osteogenic differentiation depends on direct or close cell contact. BMSCs were cultured in the presence of EC CM, in the presence of media shared with ECs, on an ECM that was laid down by ECs, and in coculture with ECs in which there was cell-cell contact between the two cell types. Only in the condition in which the cells 
were in direct contact was a consistent enhancement in osteogenic differentiation of BMSCs, as measured by ALP activity and OCN production from BMSCs, observed. Further, this enhancement in differentiation was also shown to be specific to ECs and not due to nonspecific BMSC interactions with other cell types, as demonstrated with the control conditions involving coculture of BMSCs with HFFs and HGFs. Because neither CM nor ECM from ECs increased BMSC differentiation, these findings suggest that ECs require direct cell contact with BMSCs for their BMP-2 production to be effective. This finding is supported by a previous report that osteoblastic progenitor cells derived from bone marrow increased ALP activity when in direct contact with HUVECs, and this effect was mediated by gap junctional communication between the two cell types (8).

A key finding from our studies was the functional significance of BMP-2 expression from ECs. Past studies demonstrated BMP-2 expression from ECs $(9,10,28)$, although the effect of this EC BMP-2 production in osteogenic differentiation was not evaluated. To elucidate the role of BMP2 in the coculture system used in the present studies, its production in ECs was inhibited. This inhibition resulted in a significant decrease in osteogenic differentiation of BMSCs cocultured with ECs relative to coculture with ECs whose BMP-2 production was not inhibited. Cell-cell contact and the manner in which these two cell types associate with one another may mediate the BMP-2 signaling. Addition of $100 \mathrm{ng} / \mathrm{ml}$ recombinant human BMP-2 (rhBMP-2) directly to BMSC cultures resulted in ALP activity that mimicked that of BMSCs cultured with ECs. Analysis of EC BMP-2 production by Western blot analysis revealed that $\sim 50 \mathrm{ng}$ BMP-2 was produced by the number of ECs used in coculture studies. Thus, EC-derived BMP-2 had a significant effect on BMSCs at a lower level than did direct addition of rhBMP-2 to BMSCs. Possible reasons for this include that ECs provide additional signals to BMSCs that act in concert with the BMP-2; EC-derived BMP-2 is more potent than rhBMP-2; and EC-derived BMP-2 is presented differently to BMSCs than is the rhBMP-2.

Not only did ECs enhance osteogenic differentiation of BMSCs in vitro, but cell transplantation studies demonstrated that they also enhanced the bone-forming ability of BMSCs in vivo. Cell transplantation on polymer scaffolds has become a popular approach in strategies to engineer bone tissue, and a number of studies have utilized bone marrow-derived cells to this end (20, 2933). In many instances, osteogenesis occurred within weeks of implantation, but bone development could be limited by the lack of vascular supply to the developing tissue. To address this problem, strategies have been developed that aim to enhance angiogenesis in bone regeneration applications (34-37), through the use of vascular pedicles or delivery of an angiogenic growth factor from the scaffold (38). There have not been any reports of EC transplantation being used as a strategy to enhance vascularization of tissue engineered bone, although EC transplantation on polymer scaffolds has been shown to result in formation of functional donor-derived vessels and a heightened angiogenic response $(39,40)$.

It is interesting that no significant difference was found in the total angiogenic response of cotransplantation of ECs with BMSCs compared with transplantation of BMSCs alone, yet blood vessel densities in both conditions increased over time. The only significant difference in neovascularization between the groups was that transplanting ECs led to an increased density of human cell-derived vessels at both time points at which blood vessel analyses were performed, which confirmed the ability of transplanted ECs to survive and form functional new vessels with the host vasculature. A small, but measurable, number of human cell-derived blood vessels were 
also found with BMSC transplantation alone, but these vessels regressed with time. BMSCs were previously reported to have the ability to form blood vessels $(40,41)$. Thus, a combination of the relatively low numbers of transplanted ECs in these studies, and the ability of BMSCs to promote neovascularization through their production of angiogenic factors $(39,41)$, likely underlies the lack of a net increase in blood vessel density with EC transplantation.

Although transplanted ECs did not significantly heighten the total angiogenic response, they increased bone tissue formation. This result suggests that the transplanted ECs had direct effects on BMSCs' ability to form bone, rather than indirect effects on bone formation through a heightened angiogenic response. The in vitro studies demonstrated the ECs used in these studies require close or direct cell contact to enhance BMSC osteogenic potential. The proximity of these cell types to one another when seeded onto PLGA scaffolds lends itself to cell-cell interactions and communication between ECs and BMSCs, which could contribute to the potentiation of the bone-forming ability of BMSCs. In addition, BMP-2 expression increases in ECs under hypoxic conditions (10), and a hypoxic environment likely exists after transplantation of ECs with BMSCs. This probably enhances the effect of EC BMP-2 production on bone formation.

Thus, this work showed that ECs produce a potent bone morphogen that affects osteogenic differentiation of BMSCs, so that the function of ECs is expanded beyond their traditional role as structural components of blood vessels. In another recent study, ECs were shown to regulate neural stem cell self-renewal, proliferation, and differentiation into neurons in vitro (42). Because of the proximity and intimate association of ECs with cells of all tissues, they may prove to provide instructive signals for a number of other different cell types and tissues as well. Further, in the context of bone and blood vessel formation, the intricate interaction between BMSCs and ECs may have the potential to be exploited in tissue-engineering applications for the therapeutic regeneration of bone.

\section{ACKNOWLEDGMENTS}

We would like to thank John Baker (Orthopaedic Research Laboratories, University of Michigan) and Chris Strayhorn (School of Dentistry, University of Michigan) for providing histology support. The authors are grateful to the National Institute of Dental and Craniofacial Research for funding of an Individual Pre-doctoral Dental Scientist Fellowship (F30 DE05747) to D. K., and for research funding to the laboratories of P. K. (RO1-DE013835). This work was supported by the U.S. Army Research Laboratory and the U.S. Army Research Office under grant DAAD190310168.

\section{REFERENCES}

1. Gerber, H. P., Vu, T. H., Ryan, A. M., Kowalski, J., Werb, Z., and Ferrara, N. (1999) VEGF couples hypertrophic cartilage remodeling, ossification and angiogenesis during endochondral bone formation. Nat. Med. 5, 623-628

2. Zaidi, M., Alam, A. S., Bax, B. E., Shankar, V. S., Bax, C. M., Gill, J. S., Pazianas, M., Huang, C. L., Sahinoglu, T., Moonga, B. S., et al. (1993) Role of the endothelial cell in osteoclast control: new perspectives. Bone 14, 97-102 
3. Michiels, C., De Leener, F., Arnould, T., Dieu, M., and Remacle, J. (1994) Hypoxia stimulates human endothelial cells to release smooth muscle cell mitogens: role of prostaglandins and bFGF. Exp. Cell Res. 213, 43-54

4. Streeten, E. A., and Brandi, M. L. (1990) Biology of bone endothelial cells. Bone Miner. 10, $85-94$

5. Gerritsen, M. E., and Bloor, C. M. (1993) Endothelial cell gene expression in response to injury. FASEB J. 7, 523-532

6. Jones, A. R., Clark, C. C., and Brighton, C. T. (1995) Microvessel endothelial cells and pericytes increase proliferation and repress osteoblast phenotypic markers in rat calvarial bone cell cultures. J. Orthop. Res. 13, 553-561

7. Villanueva, J. E., and Nimni, M. E. (1990) Promotion of calvarial cell osteogenesis by endothelial cells. J. Bone Miner. Res. 5, 733-739

8. Villars, F., Guillotin, B., Amedee, T., Dutoya, S., Bordenave, L., Bareille, R., and Amedee, J. (2002) Effect of HUVEC on human osteoprogenitor cell differentiation needs heterotypic gap junction communication. Am. J. Physiol. 282, C775-C785

9. Li, W. M., Huang, W. Q., Huang, Y. H., Jiang, D. Z., and Wang, Q. R. (2000) Positive and negative hematopoietic cytokines produced by bone marrow endothelial cells. Cytokine 12, $1017-1023$

10. Bouletreau, P. J., Warren, S. M., Spector, J. A., Peled, Z. M., Gerrets, R. P., Greenwald, J. A., and Longaker, M. T. (2002) Hypoxia and VEGF up-regulate BMP-2 mRNA and protein expression in microvascular endothelial cells: implications for fracture healing. Plast. Reconstr. Surg. 109, 2384-2397

11. Friedenstein, A. J., Ivanov-Smolenski, A. A., Chajlakjan, R. K., Gorskaya, U. F., Kuralesova, A. I., Latzinik, N. W., and Gerasimow, U. W. (1978) Origin of bone marrow stromal mechanocytes in radiochimeras and heterotopic transplants. Exp. Hematol. 6, 440444

12. Latsinik, N. V., Gorskaia Iu, F., Grosheva, A. G., Domogatskii, S. P., and Kuznetsov, S. A. (1986) [The stromal colony-forming cell (CFUf) count in the bone marrow of mice and the clonal nature of the fibroblast colonies they form.] Ontogenez 17, 27-36 [In Russian]

13. Owen, M. (1988) Marrow stromal stem cells. J. Cell Sci. Suppl. 10, 63-76

14. Patt, H. M., Maloney, M. A., and Flannery, M. L. (1982) Hematopoietic microenvironment transfer by stromal fibroblasts derived from bone marrow varying in cellularity. Exp. Hematol. 10, 738-742

15. Ashton, B. A., Allen, T. D., Howlett, C. R., Eaglesom, C. C., Hattori, A., and Owen, M. (1980) Formation of bone and cartilage by marrow stromal cells in diffusion chambers in vivo. Clin. Orthop. (151), 294-307 
16. Krebsbach, P. H., Gu, K., Franceschi, R. T., and Rutherford, R. B. (2000) Gene therapydirected osteogenesis: BMP-7-transduced human fibroblasts form bone in vivo. Hum. Gene Ther. 11, 1201-1210

17. Shang, Q., Wang, Z., Liu, W., Shi, Y., Cui, L., and Cao, Y. (2001) Tissue-engineered bone repair of sheep cranial defects with autologous bone marrow stromal cells. J. Craniofac. Surg. 12:586-593; discussion 594-595.

18. Kon, E., Muraglia, A., Corsi, A., Bianco, P., Marcacci, M., Martin, I., Boyde, A., Ruspantini, I., Chistolini, P., Rocca, M., et al. (2000) Autologous bone marrow stromal cells loaded onto porous hydroxyapatite ceramic accelerate bone repair in critical-size defects of sheep long bones. J. Biomed. Mater. Res. 49, 328-337

19. Gysin, R., Wergedal, J. E., Sheng, M. H., Kasukawa, Y., Miyakoshi, N., Chen, S. T., Peng, H., Lau, K. H., Mohan, S., and Baylink, D. J. (2002) Ex vivo gene therapy with stromal cells transduced with a retroviral vector containing the BMP4 gene completely heals critical size calvarial defect in rats. Gene Ther. 9, 991-999

20. Krebsbach, P. H., Kuznetsov, S. A., Satomura, K., Emmons, R. V., Rowe, D. W., and Robey, P. G. (1997) Bone formation in vivo: comparison of osteogenesis by transplanted mouse and human marrow stromal fibroblasts. Transplantation 63, 1059-1069

21. Krebsbach, P. H., Kuznetsov, S. A., Bianco, P., and Robey, P. G. (1999) Bone marrow stromal cells: characterization and clinical application. Crit. Rev. Oral Biol. Med. 10, 165181

22. Manolagas, S. C., Burton, D. W., and Deftos, L. J. (1981) 1,25-Dihydroxyvitamin $\mathrm{D}_{3}$ stimulates the alkaline phosphatase activity of osteoblast-like cells. J. Biol. Chem. 256, 7115-7117

23. D'Ippolito, G., Schiller, P. C., Ricordi, C., Roos, B. A., and Howard, G. A. (1999) Agerelated osteogenic potential of mesenchymal stromal stem cells from human vertebral bone marrow. J. Bone Miner. Res. 14, 1115-1122

24. Cesarone, C. F., Bolognesi, C., and Santi, L. (1979) Improved microfluorometric DNA determination in biological material using 33258 Hoechst. Anal. Biochem. 100, 188-197

25. Mooney, D. J., Baldwin, D. F., Suh, N. P., Vacanti, J. P., and Langer, R. (1996) Novel approach to fabricate porous sponges of poly(D,L-lactic-co-glycolic acid) without the use of organic solvents. Biomaterials 17, 1417-1422

26. Nor, J. E., Peters, M. C., Christensen, J. B., Sutorik, M. M., Linn, S., Khan, M. K., Addison, C. L., Mooney, D. J., and Polverini, P. J. (2001) Engineering and characterization of functional human microvessels in immunodeficient mice. Lab. Invest. 81, 453-463

27. Bronckers, A. L., Gay, S., Finkelman, R. D., and Butler, W. T. (1987) Immunolocalization of Gla proteins (osteocalcin) in rat tooth germs: comparison between indirect 
immunofluorescence, peroxidase-antiperoxidase, avidin-biotin-peroxidase complex, and avidin-biotin-gold complex with silver enhancement. J. Histochem. Cytochem. 35, 825-830

28. Mohan, R. R., Kim, W. J., Chen, L., and Wilson, S. E. (1998) Bone morphogenic proteins 2 and 4 and their receptors in the adult human cornea. Invest. Ophthalmol. Vis. Sci. 39, 26262636

29. Mendes, S. C., Tibbe, J. M., Veenhof, M., Bakker, K., Both, S., Platenburg, P. P., Oner, F. C., De Bruijn, J. D., and Van Blitterswijk, C. A. (2002) Bone tissue-engineered implants using human bone marrow stromal cells: effect of culture conditions and donor age. Tissue Eng. 8, 911-920

30. Dong, J., Kojima, H., Uemura, T., Kikuchi, M., Tateishi, T., and Tanaka, J. (2001) In vivo evaluation of a novel porous hydroxyapatite to sustain osteogenesis of transplanted bone marrow-derived osteoblastic cells. J. Biomed. Mater. Res. 57, 208-216

31. Gao, J., Dennis, J. E., Solchaga, L. A., Awadallah, A. S., Goldberg, V. M., and Caplan, A. I. (2001) Tissue-engineered fabrication of an osteochondral composite graft using rat bone marrow-derived mesenchymal stem cells. Tissue Eng. 7, 363-371

32. Ohgushi, H., Okumura, M., Tamai, S., Shors, E. C., and Caplan, A. I. (1990) Marrow cell induced osteogenesis in porous hydroxyapatite and tricalcium phosphate: a comparative histomorphometric study of ectopic bone formation. J. Biomed. Mater. Res. 24, 1563-1570

33. Okumura, M., Ohgushi, H., and Tamai, S. (1991) Bonding osteogenesis in coralline hydroxyapatite combined with bone marrow cells. Biomaterials 12, 411-416

34. Pelissier, P., Villars, F., Mathoulin-Pelissier, S., Bareille, R., Lafage-Proust, M. H., and Vilamitjana-Amedee, J. (2003) Influences of vascularization and osteogenic cells on heterotopic bone formation within a madreporic ceramic in rats. Plast. Reconstr. Surg. 111, 1932-1941

35. Vogelin, M. D. E., Jones, N. F., Lieberman, J. R., Baker, J. M., Tsingotjidou, A. S., and Brekke, J. H. (2002) Prefabrication of bone by use of a vascularized periosteal flap and bone morphogenetic protein. Plast. Reconstr. Surg. 109, 190-198

36. Yajima, H., Tamai, S., Ishida, H., and Kisanuki, O. (1995) Prefabricated vascularized periosteal grafts using fascial flap transfers. J. Reconstr. Microsurg. 11, 201-205

37. Romana, M. C., and Masquelet, A. C. (1990) Vascularized periosteum associated with cancellous bone graft: an experimental study. Plast. Reconstr. Surg. 85, 587-592

38. Murphy, W. L., Simmons, C. A., Kaigler, D., and Mooney, D. J. (2004) Bone regeneration via a mineral substrate and induced angiogenesis. J. Dent. Res. 83, 204-210

39. Kaigler, D., Krebsbach, P. H., Polverini, P. J., and Mooney, D. J. (2003) Role of vascular endothelial growth factor in bone marrow stromal cell modulation of endothelial cells. Tissue Eng. 9, 95-103 
40. Schechner, J. S., Nath, A. K., Zheng, L., Kluger, M. S., Hughes, C. C., Sierra-Honigmann, M. R., Lorber, M. I., Tellides, G., Kashgarian, M., Bothwell, A. L., et al. (2000) In vivo formation of complex microvessels lined by human endothelial cells in an immunodeficient mouse. Proc. Natl. Acad. Sci. USA 97, 9191-9196

41. Fuchs, S., Baffour, R., Zhou, Y. F., Shou, M., Pierre, A., Tio, F. O., Weissman, N. J., Leon, M. B., Epstein, S. E., and Kornowski, R. (2001) Transendocardial delivery of autologous bone marrow enhances collateral perfusion and regional function in pigs with chronic experimental myocardial ischemia. J. Am. Coll. Cardiol. 37, 1726-1732

42. Shen, Q., Goderie, S. K., Jin, L., Karanth, N., Sun, Y., Abramova, N., Vincent, P., Pumiglia, K., and Temple, S. (2004) Endothelial cells stimulate self-renewal and expand neurogenesis of neural stem cells. Science 304, 1338-1340

43. Urist, M. R., Lietze, A., Mizutani, H., Takagi, K., Triffitt, J. T., Amstutz, J., DeLange, R., Termine, J., and Finerman, G. A. (1982) A bovine low molecular weight bone morphogenetic protein (BMP) fraction. Clin. Orthop. (162), 219-232

44. Sampath, T. K., Coughlin, J. E., Whetstone, R. M., Banach, D., Corbett, C., Ridge, R. J., Ozkaynak, E., Oppermann, H., and Rueger, D. C. (1990) Bovine osteogenic protein is composed of dimers of OP-1 and BMP-2A, two members of the transforming growth factorbeta superfamily. J. Biol. Chem. 265, 13198-13205

45. Celeste, A. J., Iannazzi, J. A., Taylor, R. C., Hewick, R. M., Rosen, V., Wang, E. A., and Wozney, J. M. (1990) Identification of transforming growth factor beta family members present in bone-inductive protein purified from bovine bone. Proc. Natl. Acad. Sci. USA 87, 9843-9847

46. Wang, E. A., Rosen, V., Cordes, P., Hewick, R. M., Kriz, M. J., Luxenberg, D. P., Sibley, B. S., and Wozney, J. M. (1988) Purification and characterization of other distinct boneinducing factors. Proc. Natl. Acad. Sci. USA 85, 9484-9488

Received July 9, 2004; accepted December 1, 2004. 
Fig. 1

A

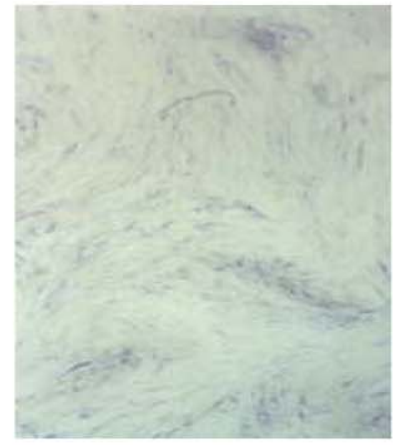

BMSCs alone

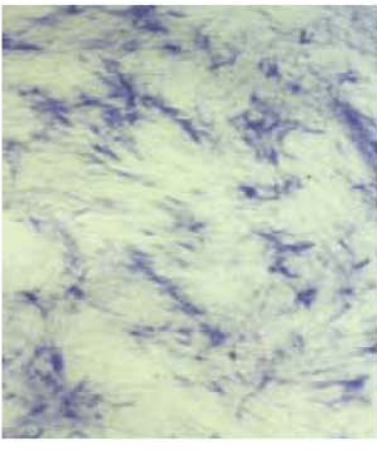

BMSCs/ECs
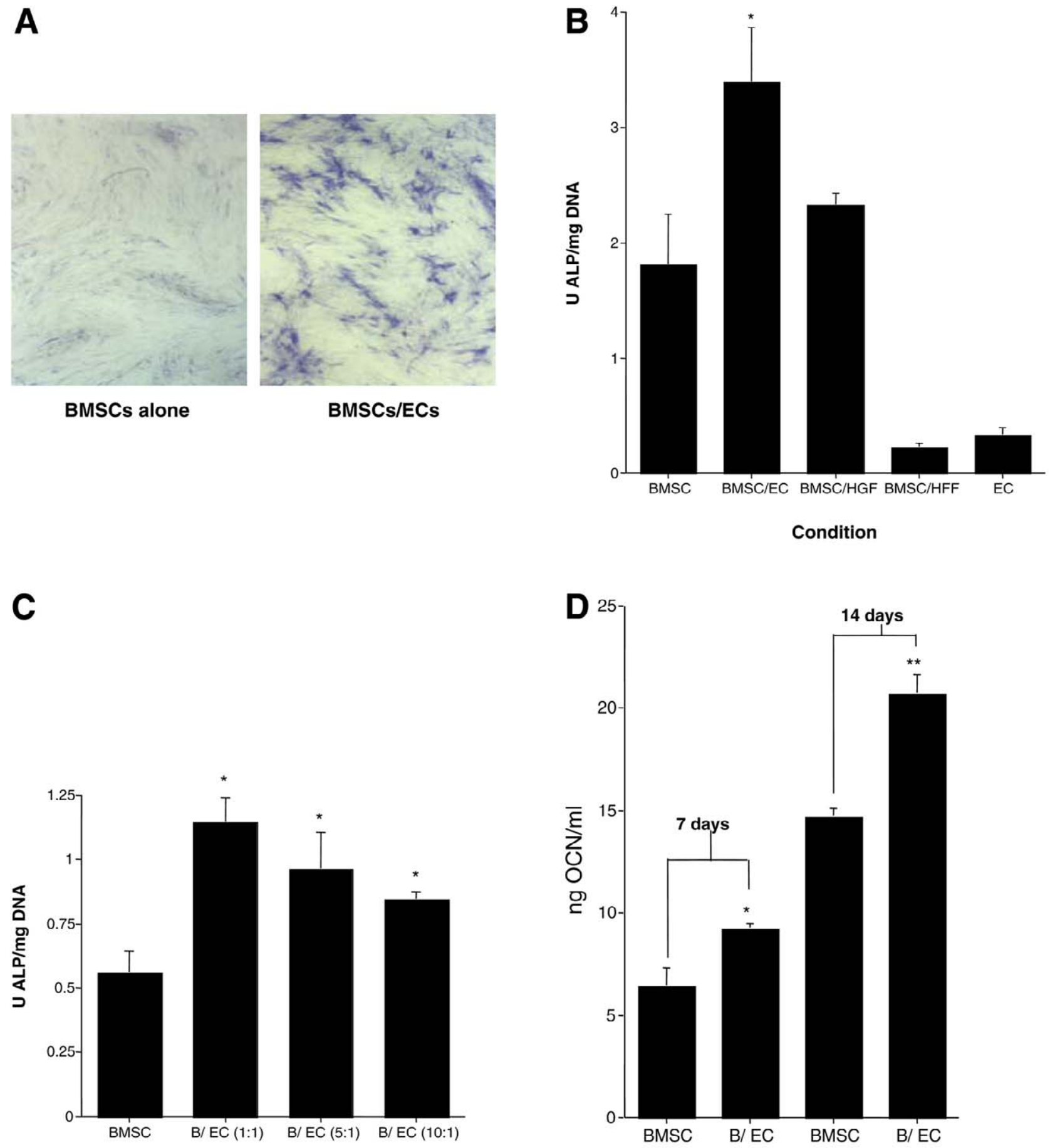

Condition

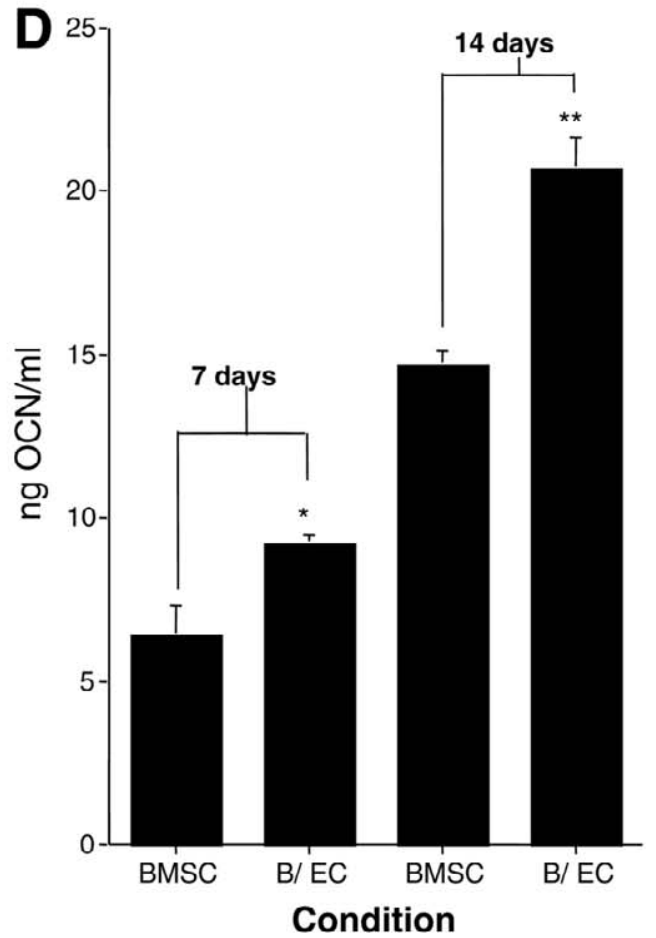

Figure 1. Influence of ECs on BMSC osteogenic differentiation. A) ALP staining of BMSCs cultured alone or cocultured with ECs after $2 \mathrm{wk}$. ALP expression was higher in cocultures, with a different pattern of staining. Original magnification $\times 200$. B $)$ Quantitative analysis of ALP expression from BMSCs cultured for 2 wk alone or cocultured at 1:1 ratios with ECs, HGF, and HFF. ALP expression was also measured for ECs grown alone in culture. $C$ ) ALP expression was measured from BMSCs cultured for $1 \mathrm{wk}$ alone and with different ratios of ECs relative to BMSCs (B). Ratios of BMSCs to ECs are in parentheses. D) OCN production from BMSCs was measured from BMSC-conditioned medium after 7 and 14 days. BMSCs were cultured alone or were cocultured with ECs. OCN from ECs alone was also measured, but no detectable levels were present in EC CM. Values represent means $\pm \operatorname{SE}(n=3)$. $* P<0.05$, compared with the BMSC condition; $* * P<0.05$, compared with BMSC 14-day time point for OCN ELISA. 
Fig. 2

A

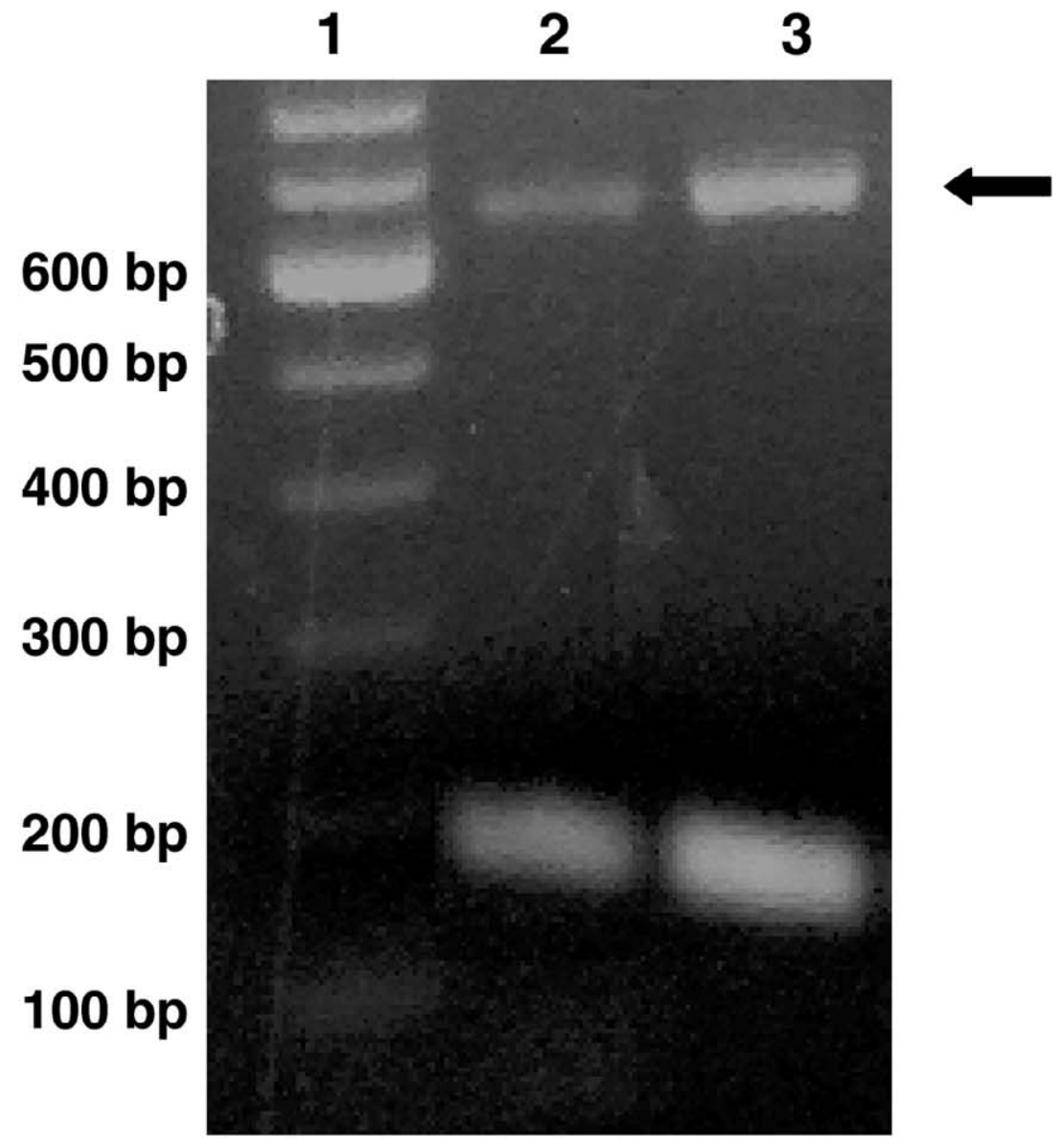

1
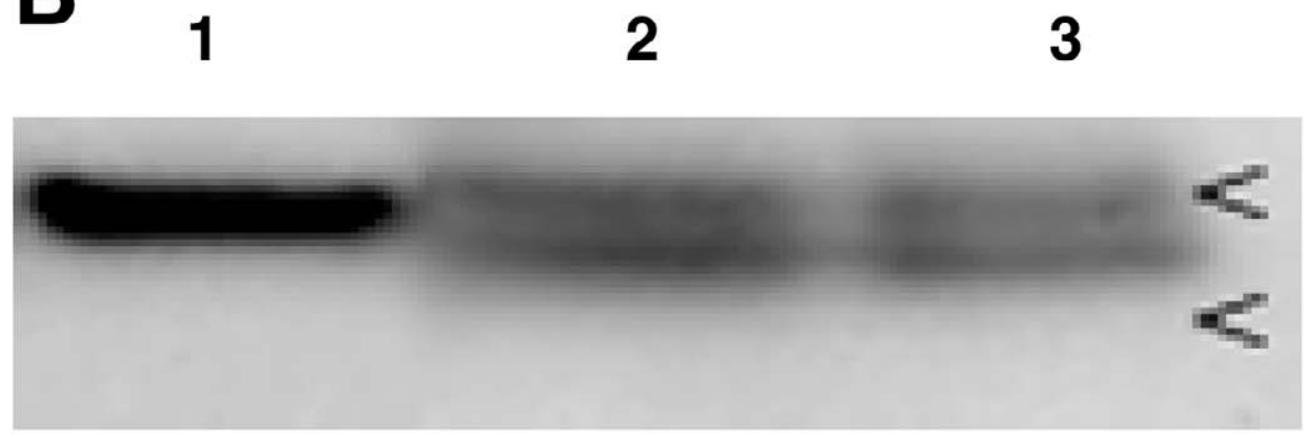

\section{$18 \mathrm{kDa}$}

8.5 kDa

Figure 2. BMP-2 expression in ECs. $A$ ) RNA was isolated from ECs and analyzed by RT-PCR for expression of BMP-2. HDMEC (lane 2) and HUVEC (lane 3) cDNAs were used as templates for BMP-2 forward and reverse primers (arrow), and rRNA primers (control RNA); lane 1 contains a 100-bp DNA ladder. $\boldsymbol{B}$ ) Cell lysates were collected from HDMECs and HUVECs, and Western blotting was performed to detect production of BMP-2. Lane 1 was loaded with $50 \mathrm{ng}$ of recombinant human BMP-2, lane 2 contained HDMEC lysates, and lane 3 contained HUVEC lysates. BMP-2 is a homodimer in its native form and migrates at about $36 \mathrm{kDa}$. However, it is not uncommon after reduction of the molecule to see double banding (at $18 \mathrm{kDa}$ ) in Western blots because of differences in the stage of glycosylation of the immature form of the protein (43-46). 
Fig. 3

A

$$
\underline{2 \text { days }}
$$

EC-Lipo EC-siRNA

BMP-2

GAPDH

$\underline{3 \text { days }}$

\begin{tabular}{|l|l|}
\hline EC-Lipo & EC-siRNA \\
& BMP-2 \\
\hline & GAPDH \\
\hline
\end{tabular}

$\underline{4 \text { days }}$

\begin{tabular}{cc} 
EC-Lipo & EC-siRNA \\
\hline
\end{tabular}

BMP-2

GAPDH

B

BMSC + EC-siRNA

BMSC + EC-Lipo

BMSC

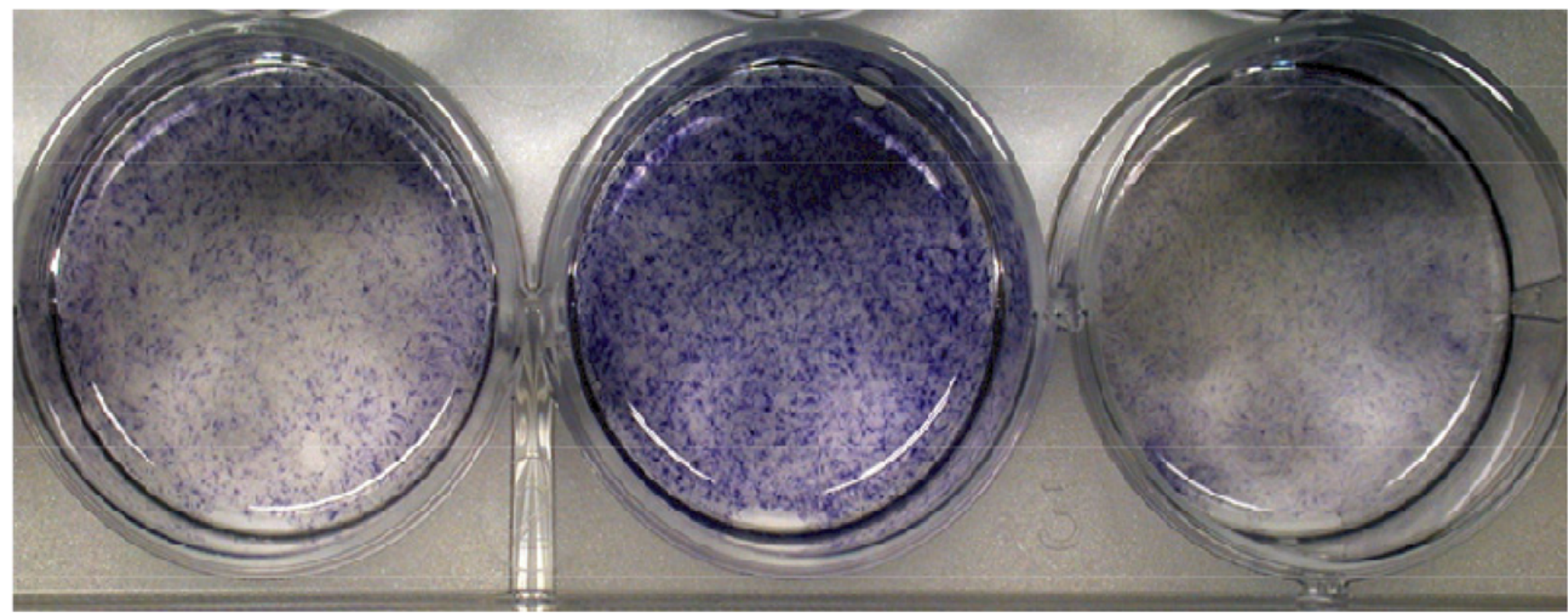


Fig. 3 (cont)

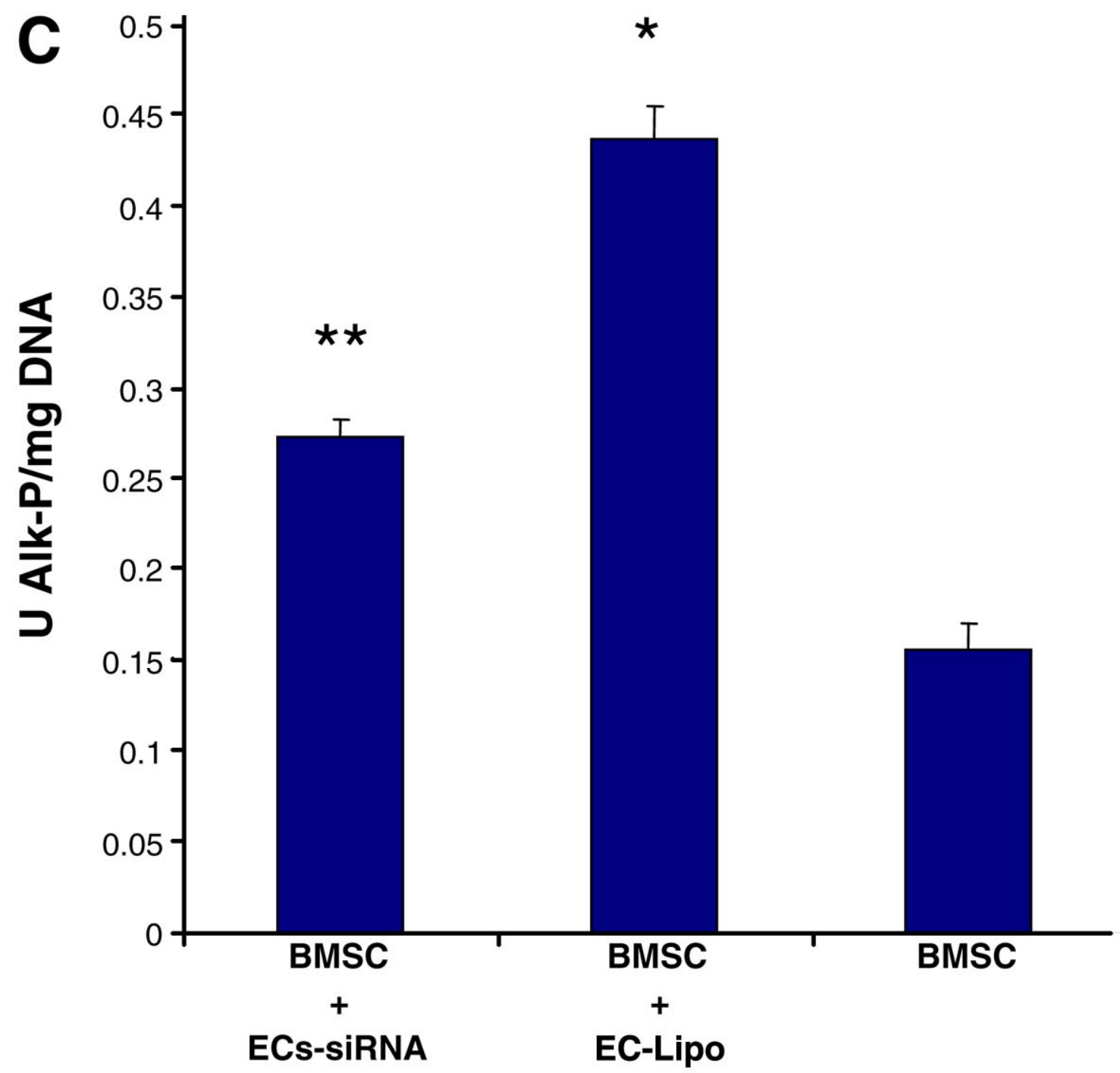

Figure 3. siRNA inhibition of BMP-2. A) BMP-2 siRNA was transfected into ECs (EC-siRNA) by using Lipofectamine 2000, and BMP-2 protein levels were measured by Western blot analysis 2, 3, and 4 days after transfection. Another group of ECs was transfected with only Lipofectamine 2000 (EC-Lipo). B) Digital photographs showing ALP staining of BMSCs cultured for 2 days with EC-siRNA, with EC-Lipo, and alone. $C$ ) ALP expression of BMSCs cultured with ECsiRNA, with EC-Lipo, and alone was measured. Values represent means $\pm \operatorname{SE}(n=3) .{ }^{*} P<0.05$, BMSC + EC-siRNA compared with BMSC + EC-Lipo; $* * P<0.05$, BMSC + EC-siRNA compared with BMSC. 
Fig. 4

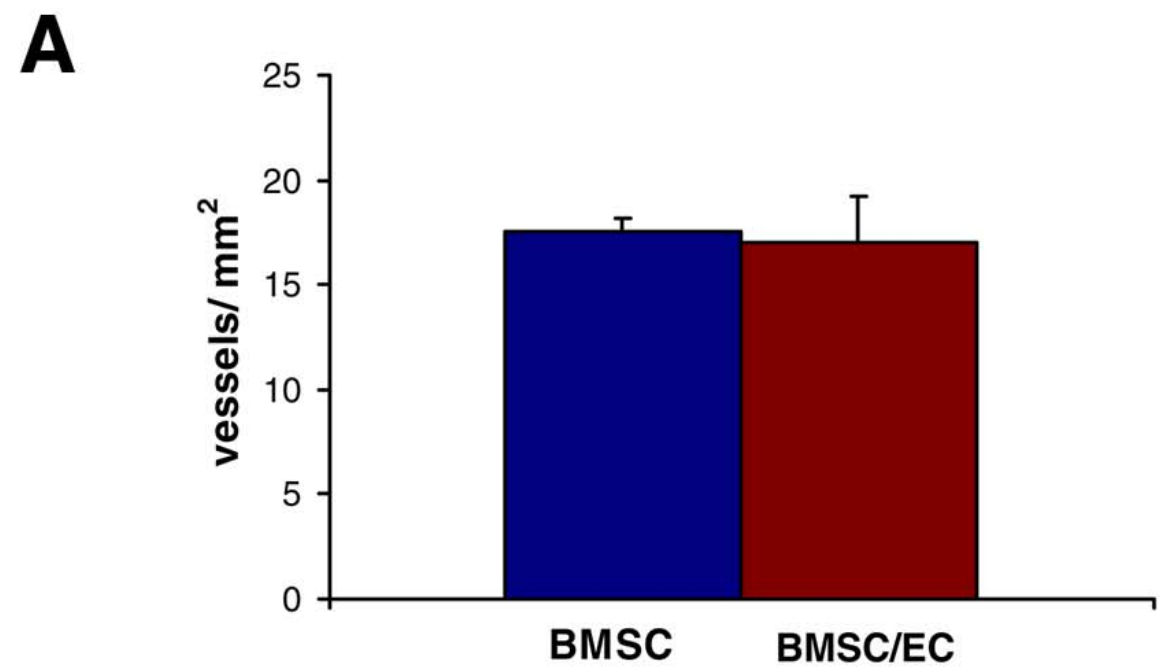

\section{B}

\section{Low mag.}
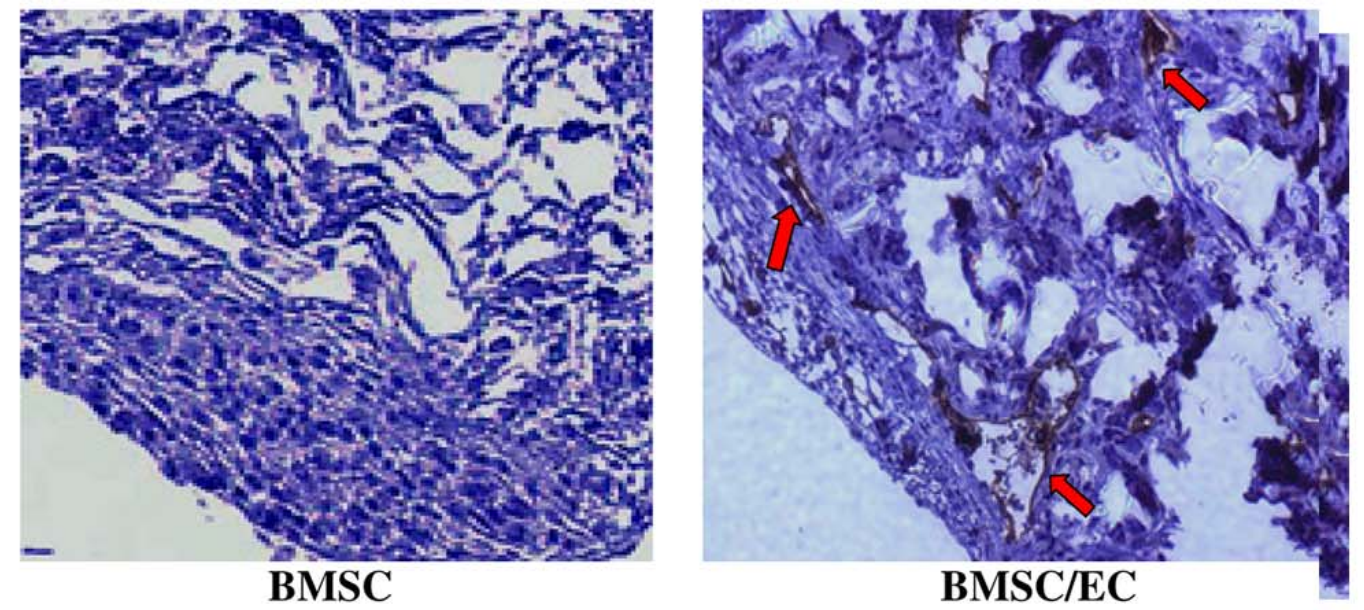

High mag. (BMSC/EC)

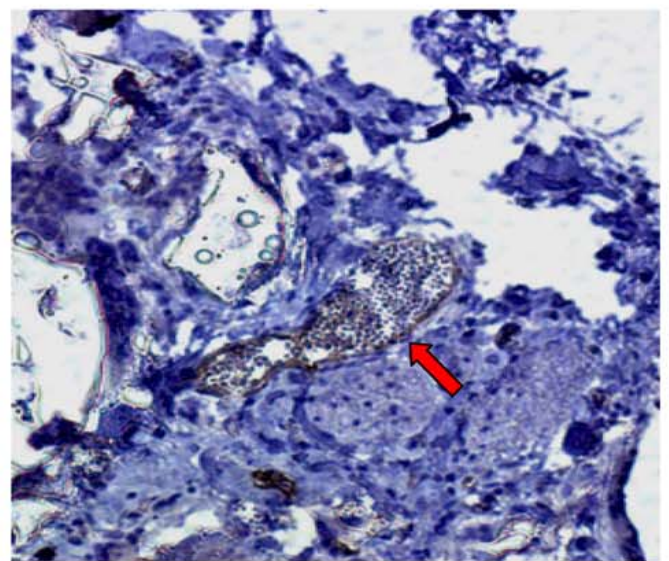


Fig. 4 (cont)

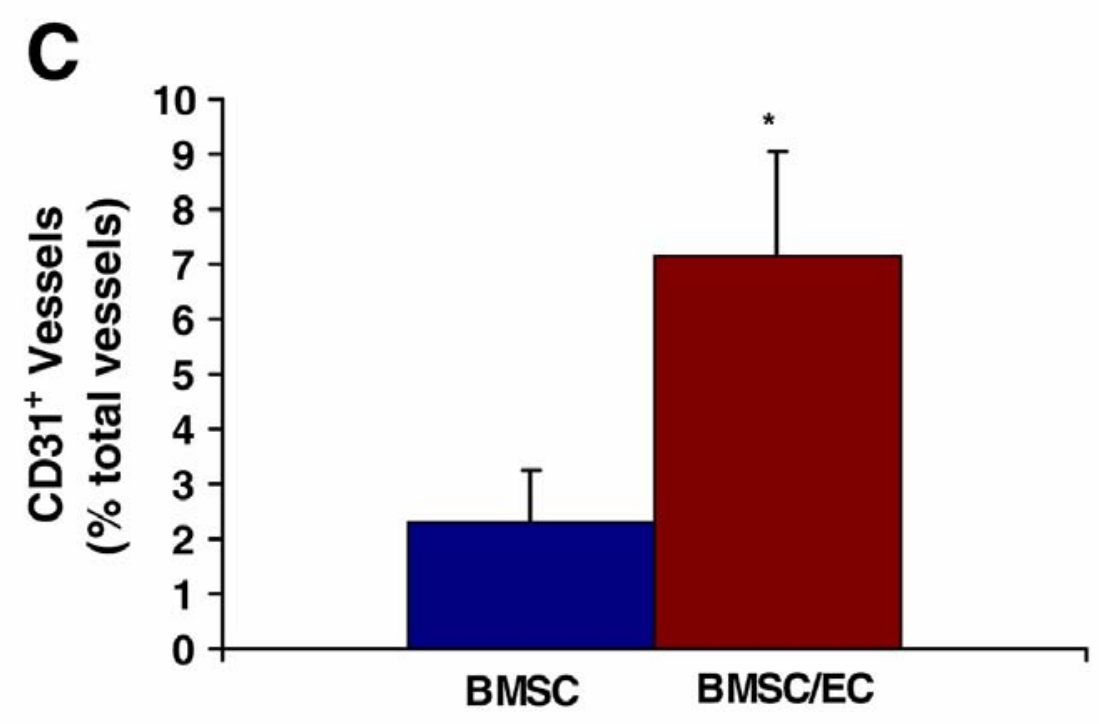

Figure 4. Blood vessel analysis of implants at 2 wk. A) In H\&E-stained sections, functional blood vessels were defined by structures that had a clearly defined lumen containing red blood cells. Vessels were counted manually at $\times 200$ magnification and normalized to tissue area. Vessels were measured in transplants containing BMSCs and in cotransplants containing BMSCs and ECs (BMSC/EC). B) Arrows point to human-derived vessels, which are clearly visible via human CD31 immunostaining (brown structures) in the condition of ECs cotransplanted with BMSCs. Low magnification (mag.) $\times 200$; higher magnification $\times 400$. C) Percentage of total functional vessels that were human derived. Values represent means $+\operatorname{SE}(n=5)$. ${ }^{*} P<0.05$. 
Fig. 5

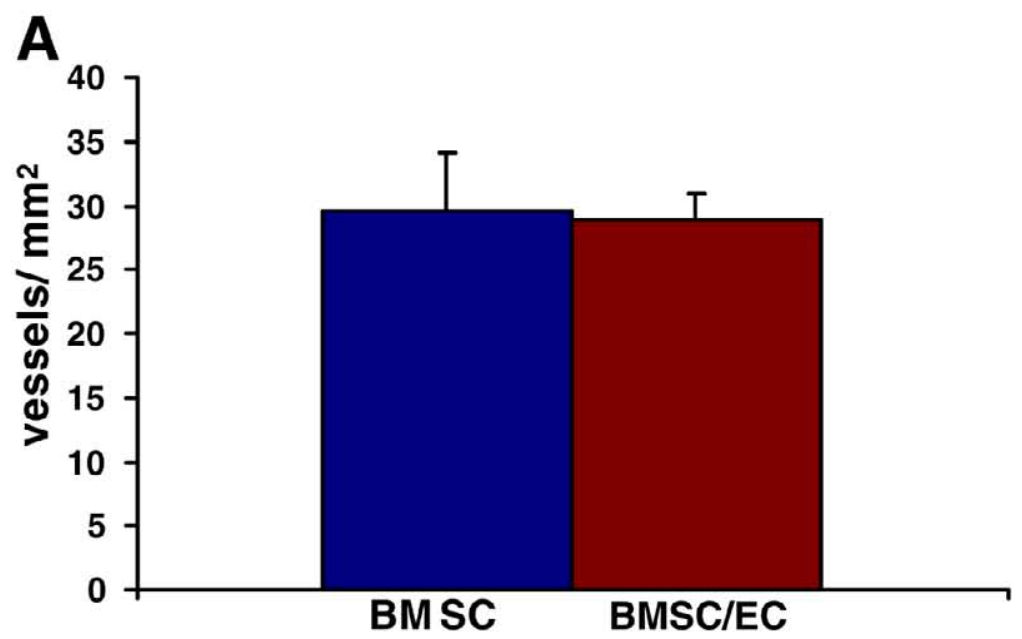

B
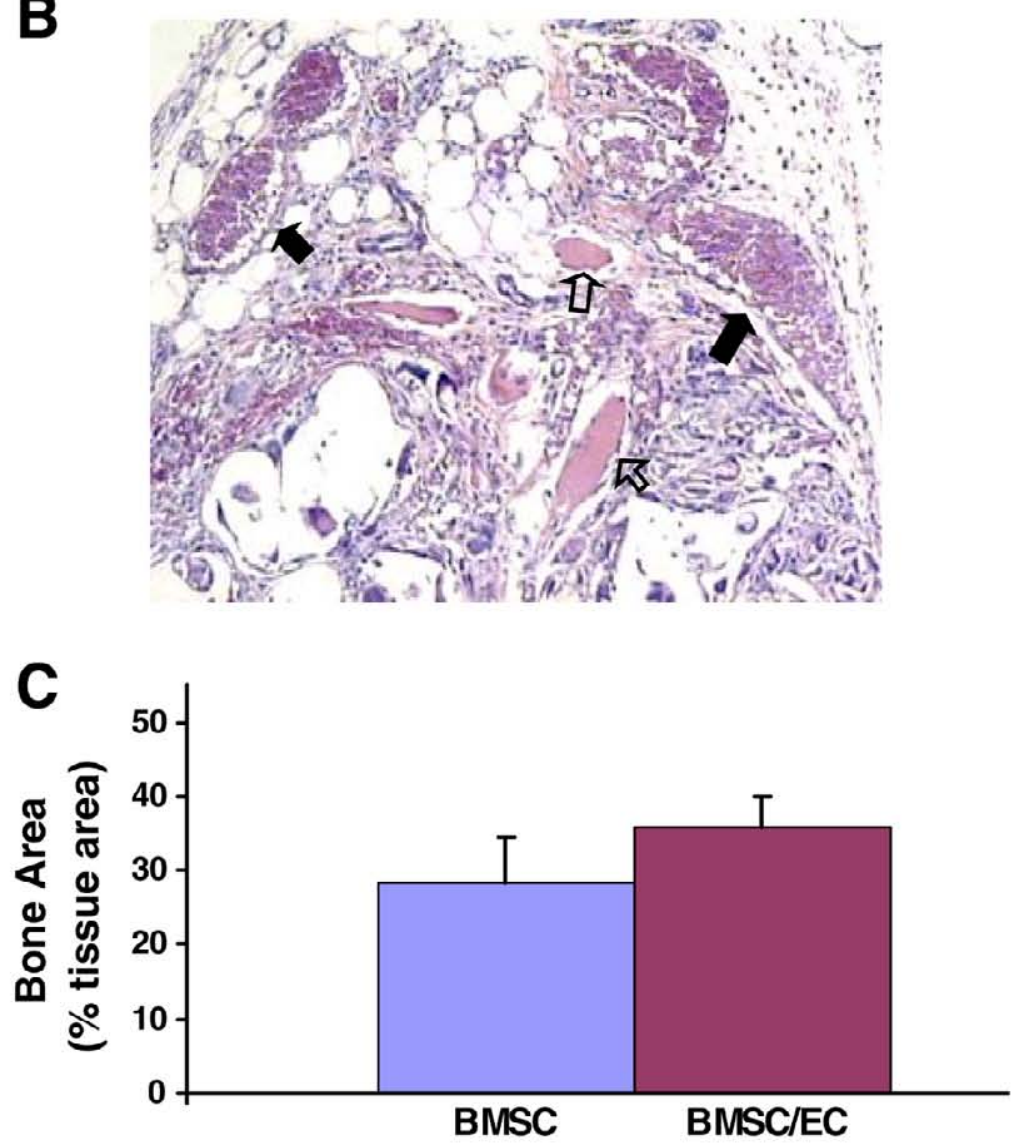

Figure 5. Blood vessel analysis and bone histomorphometry at 4 wk. A) In H\&E-stained sections, manual counts of functional vessels at $\times 200$ were done for scaffolds containing BMSC and ECs, and for scaffold containing only BMSCs. B) Photomicrograph from H\&E-stained scaffold containing only BMSCs, showing a robust angiogenic response, as indicated by large vessel structures (filled arrows). Small areas of bone foci are also present within this field (open arrows). C) Analysis of bone formed 4 wk after implantation of PLGA scaffolds containing BMSCs alone or BMSCs and ECs. In H\&E-stained sections, bone was measured as a percentage of total tissue area within the scaffold. Values represent means $\pm \operatorname{SE}(n=5)$. 
Fig. 6
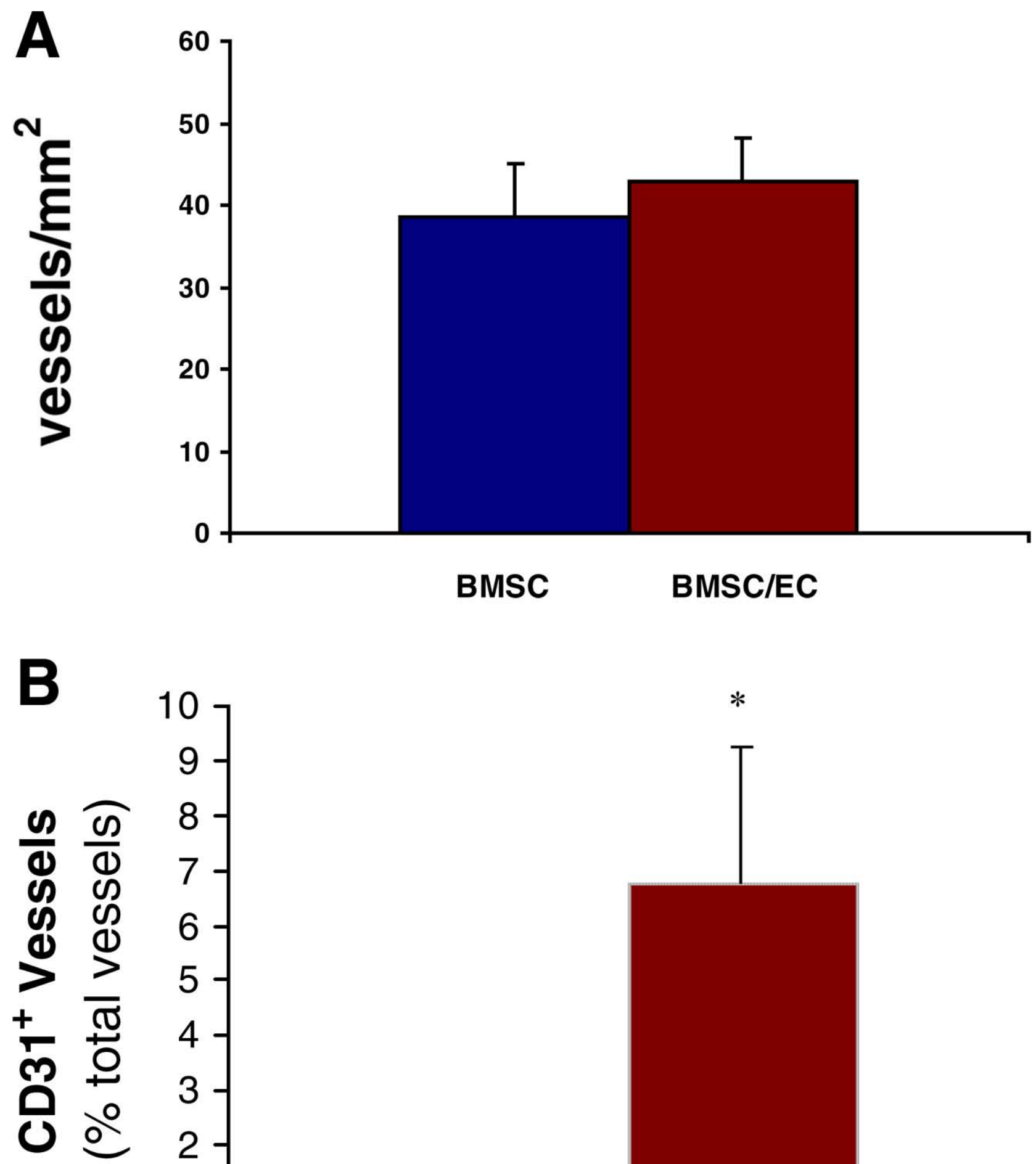

$\begin{array}{r}10 \\ 9 \\ 8 \\ 7- \\ 6- \\ 5- \\ 4- \\ 3- \\ 2- \\ 1- \\ 0\end{array}-$

$*$

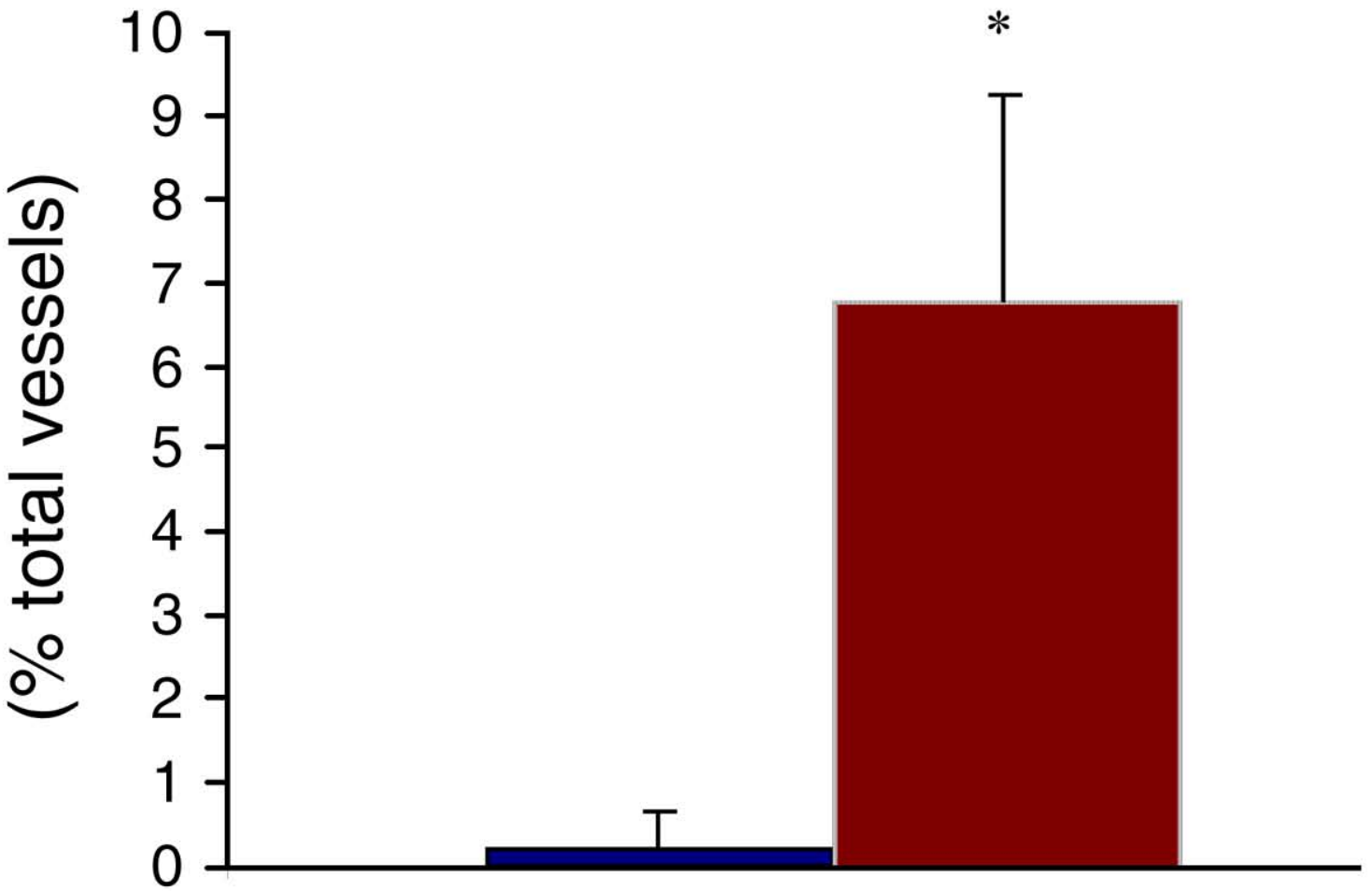

\section{BMSC BMSC/EC}

Figure 6. Angiogenesis at 8 wk. A) The total number of functional blood vessels was counted in implants retrieved 8 wk after implantation and normalized to unit area. B) Human-derived vessels were identified by vessels staining with CD31 ${ }^{+}$, and this number was plotted as a percentage of total vessels. Values represent means \pm SE. $* P<0.05$. 
Fig. 7

A

BMSC

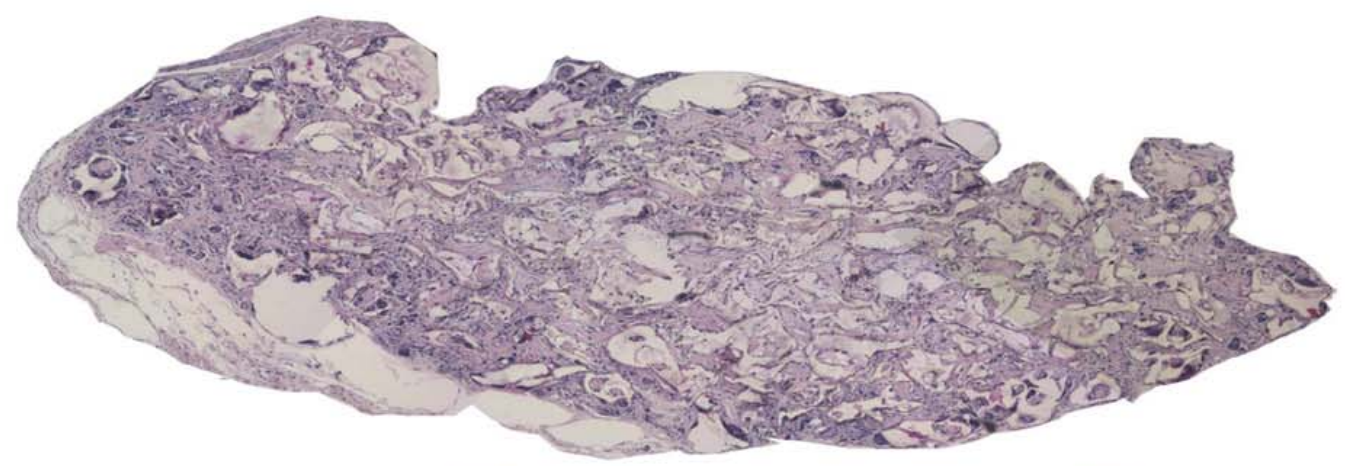

BMSC/EC
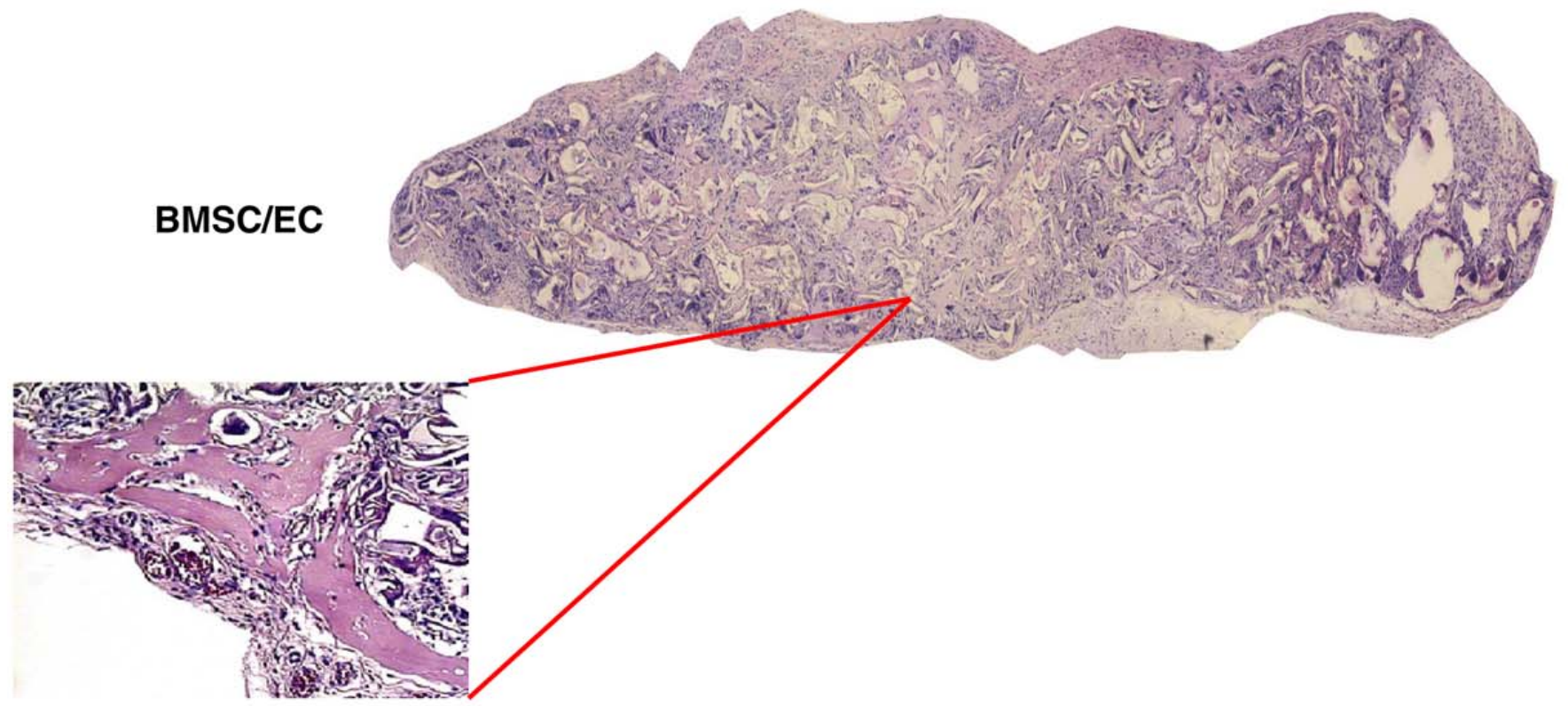

\section{B}

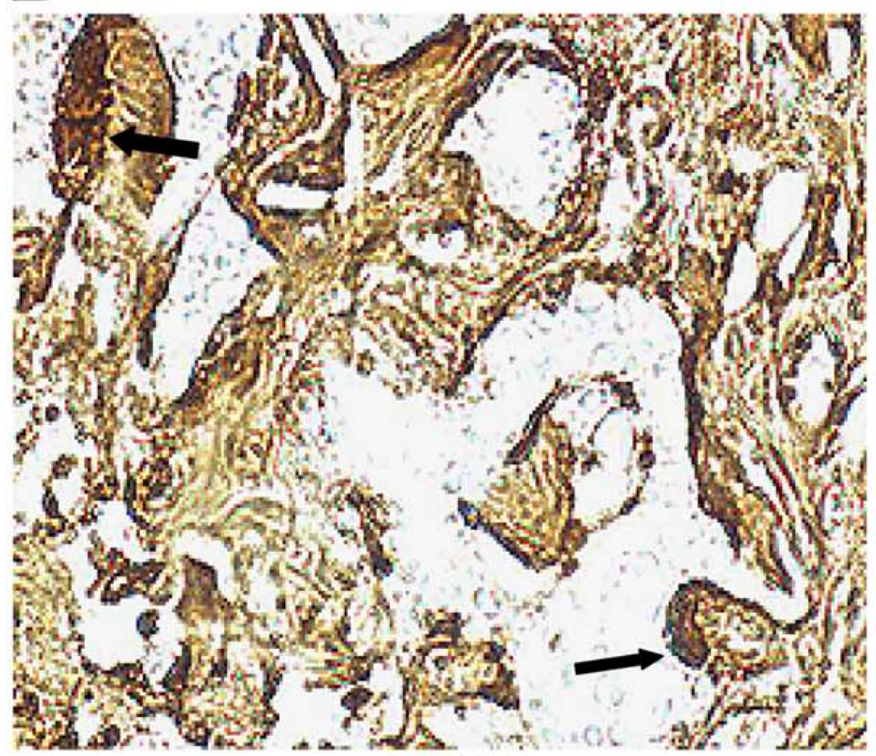

BMSC

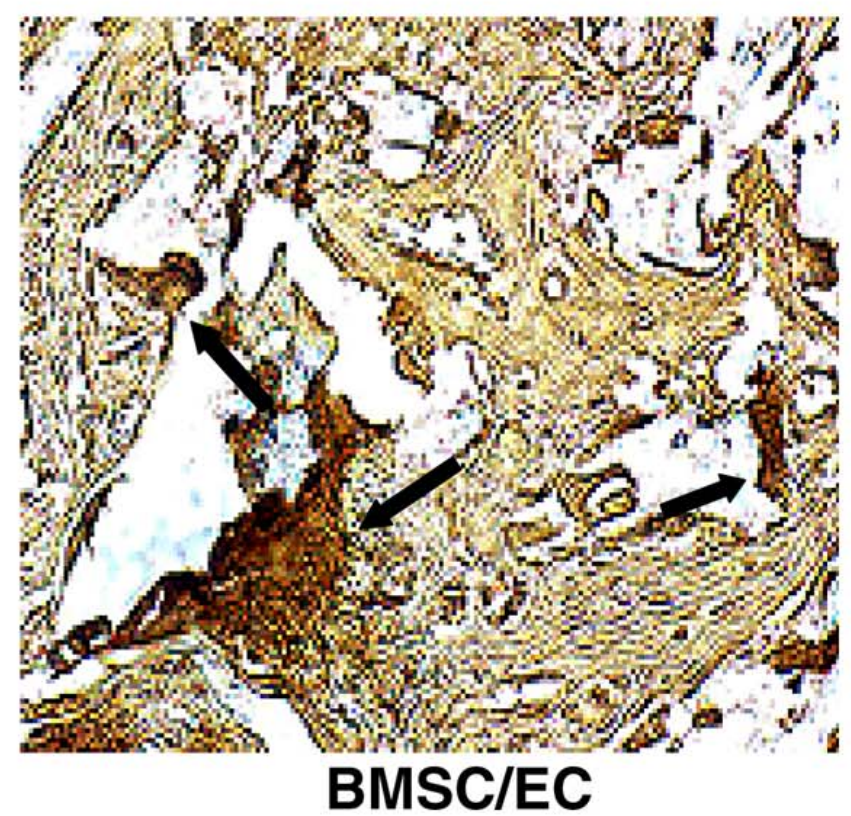


Fig. 7 (cont)

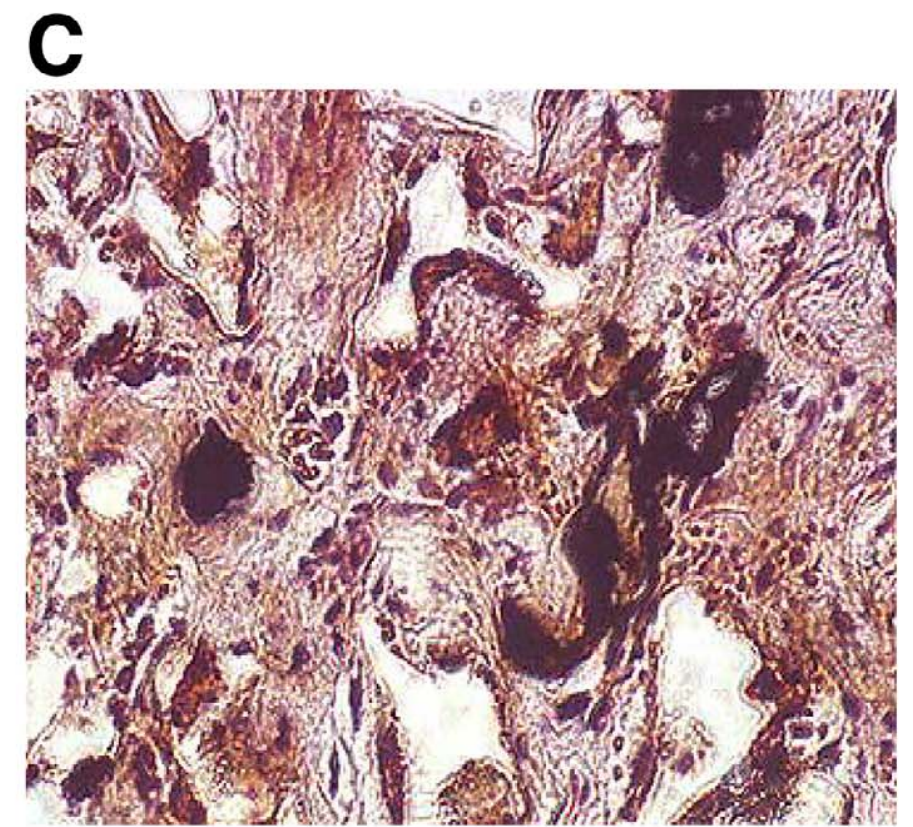

BMSC

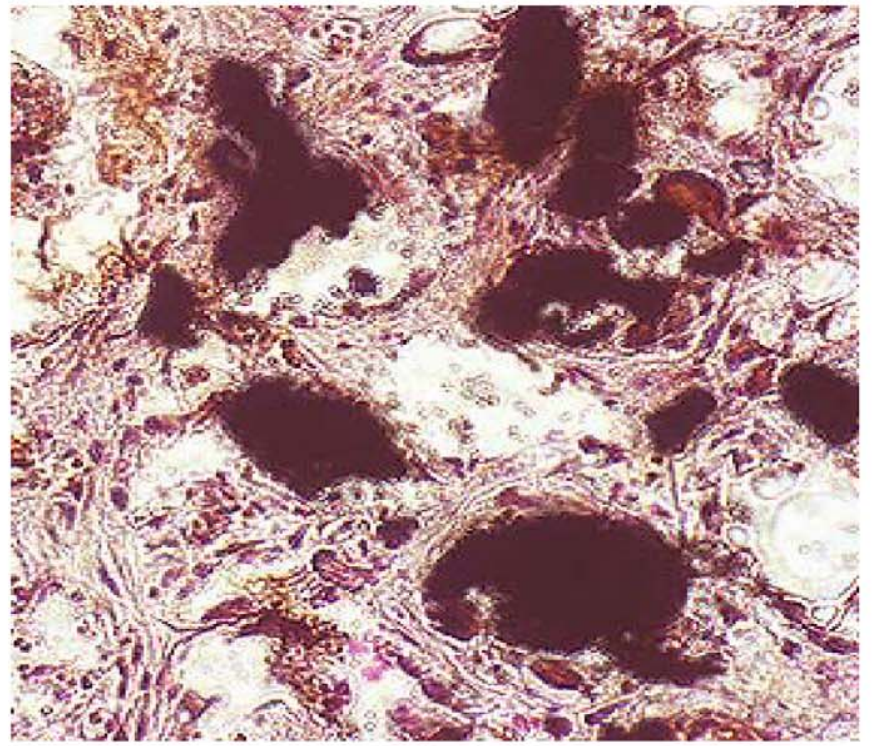

BMSC/EC

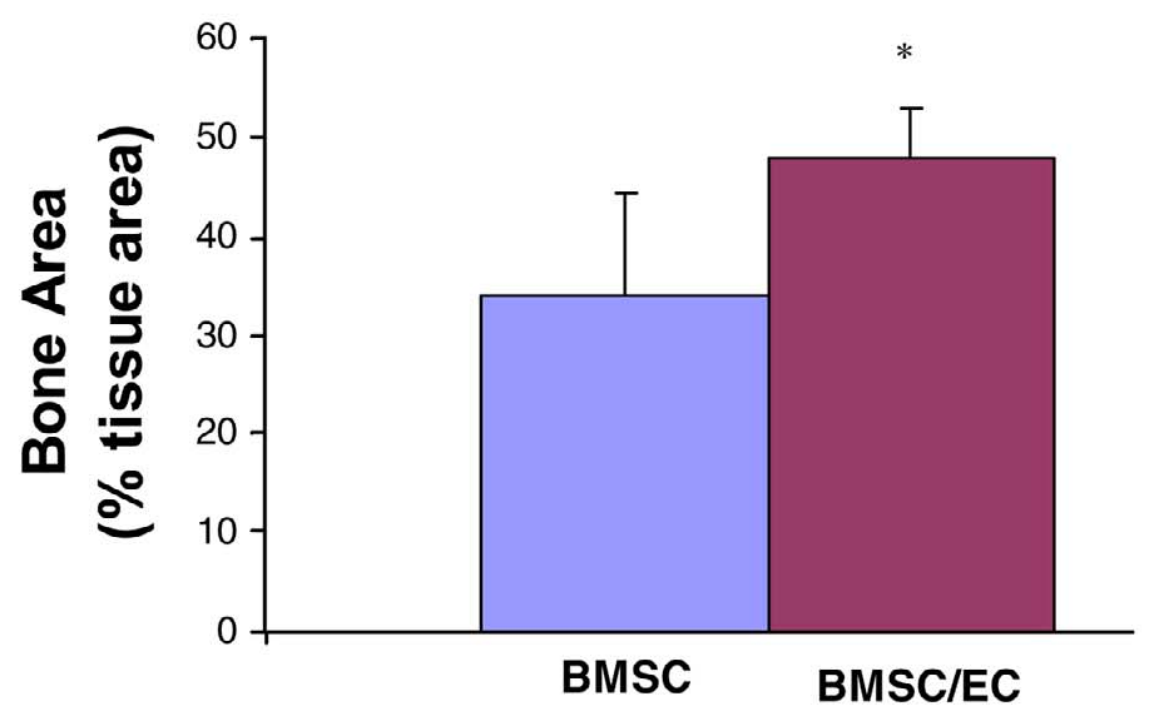

Figure 7. Bone analysis at 8 wk. A) Photomicrographs of entire scaffolds retrieved after 8 wk. Scaffolds were seeded with BMSCs alone or with ECs. High-magnification $(\times 200)$ area of bone tissue formation appears as an inset. $\boldsymbol{B})$ immunostained sections from BMSC/EC implants showing the presence of BSP (dark brown-black stain) secreted by osteoblasts and within areas of ECM (arrows). Magnification $\times 200$. C) Areas of mineralized matrix in BMSC and BMSC/EC implants as indicated by von Kossa staining (black regions). Magnification $\times 200$. $\boldsymbol{D}$ ) Bone histomorphometric analysis of scaffolds containing BMSCs alone or BMSCs and ECs retrieved 8 wk after implantation. Values represent means \pm SE $(n=5) . * P<0.05$. 